\title{
A system for the evolution of protein-protein interaction inducers
}

Jeffrey A. Dewey, Saara-Anne Azizi, Vivian Lu, Bryan C. Dickinson*

Department of Chemistry, The University of Chicago, Chicago, IL, USA.

* To whom correspondence should be addressed. Email: dickinson@uchicago.edu

Abstract: Molecules that induce interactions between proteins, often referred to as "molecular glues", are increasingly recognized as important therapeutic modalities and as entry points for rewiring cellular signaling networks. Here, we report a new PACEbased method to rapidly select and evolve molecules that mediate interactions between otherwise non-interacting proteins: rapid evolution of Protein-Protein Interaction Glues (rePPI-G). By leveraging proximity-dependent split RNA polymerase-based biosensors, we developed $E$. coli-based detection and selection systems that drive gene expression outputs only when interactions between target proteins are induced. We then validated the system using engineered bivalent molecular glues, showing that rePPI-G robustly selects for molecules that induce the target interaction. Proof-of-concept evolutions demonstrated that rePPI-G reduces the "hook" effect of the engineered molecular glues, due at least in part to tuning the interaction affinities of each individual component of the bifunctional molecule. Altogether, this work validates rePPI-G as a continuous, phagebased evolutionary technology for optimizing molecular glues, providing a strategy for developing molecules that reprogram protein-protein interactions.

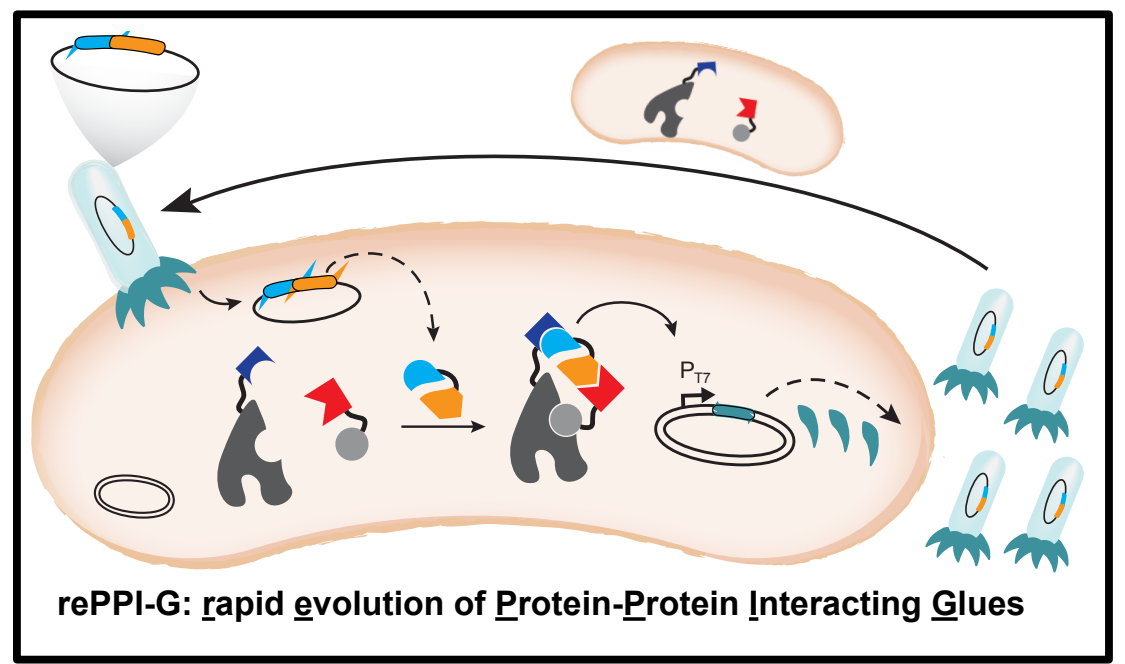

Keywords: Protein-protein interactions (PPIs), molecular glue, continuous directed evolution, PACE, protein engineering, protein interaction stabilizers 
Protein-protein interactions (PPIs) regulate nearly all cellular processes, functioning as signaling hubs, structural components, modifiers of biocatalytic complexes, and recognition motifs for intercellular communication ${ }^{1-6}$. Dysregulated PPIs have been attributed to diverse pathologies, including cancer, neurodegeneration, and autoimmunity ${ }^{7-15}$ and are therefore important therapeutic targets. PPIs are also increasingly recognized as engineerable nodes for the control of cellular information flow. Indeed, synthetic biologists have harnessed PPIs to engineer increasingly complex artificial biological systems, sensors, and materials ${ }^{16-22}$. Therefore, technologies for the generation of molecules that create or alter PPI networks have value both as therapeutic strategies and for synthetic biology applications.

While PPI inhibitors have been the focus of extensive research ${ }^{23-27}$, culminating after 30 years in the first and only FDA-approved intracellular PPI inhibitor ${ }^{28}$, molecules that instead induce interactions between proteins, i.e., "molecular glues", are garnering increased attention ${ }^{29-32}$. Approximately one hundred confirmed small molecule PPI inducers or glues, largely discovered by serendipity, currently exist, seven of which are FDA-approved for clinical use, including important medicines like rapamycin, taxol, tafamidis, and lenalidomide. However, as there are in principle at least $200,000,000$ possible PPIs among members of the human proteome, these molecules barely scratch the surface of what is possible. This potential is further illustrated by the advent of proteolysis targeting chimeras (PROTACs), bifunctional molecules that degrade proteins of interest (POls) by inducing an interaction between the $\mathrm{POI}$ and an $\mathrm{E} 3$ ubiquitin ligase ${ }^{33-39}$. While over a dozen PROTACs are entering phase I and II clinical trials, they are limited to the current pool of potent E3 ligase- and POI-binding small molecules ${ }^{40}$. Moreover, PROTACs and all bifunctional molecular glues are susceptible to the "hook effect": decreased effectiveness of the molecule at higher concentrations due to target protein saturation ${ }^{41}$. The "hook effect" is also dependent on protein target concentration, requiring screening of each new target with a panel of target- and E3 ligase-binding ligands at a wide range of concentrations.

Besides rationally designing bivalent molecules such as PROTACs, there are two other distinct molecular glue discovery methods: random screens and evolutions. Random screens, whether carried out in silico and/or in vitro, have been somewhat successful in identifying PPI inducers, particularly for the 14-3-3 protein family ${ }^{42-52}$. However, evolution has been by far the most successful method for discovering PPI inducers. There are already six evolutionarily derived FDA-approved drugs (four small molecule natural products and two bispecific antibodies) and dozens more bispecific antibodies in clinical trials ${ }^{53-56}$. However, while powerful therapeutic approaches, antibody therapies are limited to extracellular POIs, and the success of bispecific antibodies in particular can be attributed to the immune system, which itself essentially functions as a robust, modular, and rapid directed evolution method for creating POI binders. 
Directed evolution - the cyclic process of diversifying, selecting, and amplifying molecules based on activity - has generated molecules with a wide range of new activities, including metabolically altered organisms, stably expressed proteins, and selective small molecule processing enzymes ${ }^{57-64}$. In the realm of PPIs, pioneering and robust directed evolution methods such as phage, mRNA, and ribosomal display technologies have generated PPIs with subnanomolar binding affinities ${ }^{65-67}$. A range of powerful continuous evolution technologies that reduce the need for human intervention, accelerate evolution, and evolve molecules in unique biological contexts have emerged, including Phage-Assisted Continuous Evolution (PACE), Orthorep, mammalian phage assisted continuous evolution (mPACE), and viral evolution of genetically actuating sequences (VEGAS) ${ }^{68-73}$. While powerful, all of these directed evolution approaches are limited by the selection systems that can be deployed, and a robust method to evolve molecules to drive a desired intracellular PPI is lacking.

Here, we present rapid evolution of Protein-Protein Interaction Molecular $\underline{\text { Glues }}$ (rePPI-G), a new selection and continuous evolution platform for creating PPI inducers based on PACE. A powerful continuous evolution system, PACE requires linking a desired function of interest to the expression of glll, a required phage gene. We reasoned that a PACE system for evolving PPI inducers would require a biosensor system that can convert induced protein interactions into a robust and quantifiable gene expression output. To this end, we leveraged our proximity dependent split T7 RNA polymerase (RNAP) technology - which uses RNA transcription to trigger either reporter or glll gene expression - as a versatile detector of PPI inducer-based interactions in live cells ${ }^{74-76}$. The coupling of these two technologies forms rePPI-G. We validated rePPI-G using a panel of engineered bifunctional molecular glues that drive interactions between otherwise non-interacting POIs. To optimize the engineered molecular glues, we developed workflows that combined PACE-based continuous evolution with phagebased selection systems. These evolutionary approaches yielded bifunctional molecular glues with decreased "hook effect" properties, which we attribute in part to tuned interaction affinities between each half of the bifunctional molecule. Overall, this work validates rePPI-G in key proof-of-concept experiments and lays the foundation for approaches to rapidly generate molecular PPI inducers through the process of evolution. 


\section{Results}

\section{Split RNAPs can detect PPI inducers}

In previous work, we developed proximity dependent split T7 RNA polymerase (RNAP) variants that assemble into functional RNA polymerases and drive programmed gene expression outputs only when a PPI brings the two RNAP fragments together ${ }^{74}$. We demonstrated the split T7 RNAP can detect selective PPIs, light-induced dimerization events, and the presence of small molecules. Critically, we demonstrated that the split T7 RNAP system can detect validated molecular glues, including both the rapamycin-induced pairing of $\mathrm{FRB} / \mathrm{FKBP}^{74,77}$ and the abscisic acid-induced interaction of PYL/ABI ${ }^{78-79}$. Moreover, we recently developed "PhageAssisted Continuous Selection - Deep Mutational Scanning" (PACS-DMS), a method that links PPIs between a target protein and a library of binding variants to phage fitness, thereby allowing the interrogation of mutations that impact PPI formation ${ }^{80}$. Therefore, we hypothesized that the proximity dependent split RNAP system could be used to convert induced PPIs into gllI production, thus developing a new PACE system for evolving PPI inducers.

We first tested whether we could drive the assembly of two non-interacting proteins using a rationally designed bifunctional inducer molecule composed of binding partners for each target protein (Figure 1A). As a model, we selected KRas and NOXA, which have no known interaction with one another ${ }^{81-82}$. We tagged KRas with the C-terminal portion of the proximitydependent spit RNAP system (RNAP $)$ and NOXA with the N-terminal portion of the split RNAP system $\left(\right.$ RNAP $\left._{\mathrm{N}}\right)$ and tested the background assembly of the split protein using a previously established $E$. coli luciferase gene expression assay ${ }^{83}$. As expected, we observed luminescence signal comparable to the background levels of the split RNAP system itself, confirming that KRas and NOXA do not interact (Figure S1). Next, we tested whether we could promote an interaction between KRas and NOXA with a bivalent PPI inducer expressed from an IPTGinducible vector. We generated a rationally designed PPI inducer of KRas and NOXA by fusing Raf, a KRas binder, to Mcl1, a NOXA binder ${ }^{82}$. In the absence of IPTG, we observed only background levels of luminescence. However, addition of IPTG resulted in a dose-dependent enhancement of luminescence signal, with a maximum 58-fold increase in gene expression based on the engineered inducer (Figure 1B). As a control, we substituted $\mathrm{Bcl}_{\mathrm{XL}}$, a protein related to Mcl1 that does not bind $\mathrm{NOXA}^{82}$, for Mcl1 in our bivalent inducer. As expected, this control molecule did not trigger enhanced gene expression (Figure 1B). Taken together, these data confirm that the split RNAP system can detect functional bivalent PPI inducers and trigger a robust increase in gene expression.

We next sought to test the generality of the system and the ability to detect PPI inducers of varied affinities. We substituted ZA - a leucine zipper peptide which also has no known 
interaction with KRas - for NOXA in our PPI detection system. We then designed a bivalent inducer by fusing Raf to ZB, a peptide that forms a tight PPI with $Z A^{84}$. As expected, this second inducer system also triggered a robust increase in gene expression (Figure 1C). In this case, we observed gene expression activation even without IPTG induction, as well the "hook effect," with the highest concentrations of IPTG inducer causing a significant decrease in gene

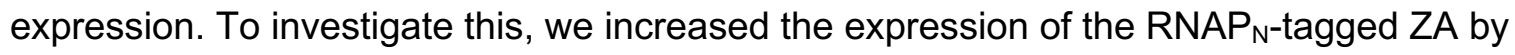
swapping the promoter from J23117 to the stronger J23114. With the stronger promoter, we observed higher levels of inducible gene expression, albeit with a more pronounced hook effect (Figure 1C). Such an effect is expected as the bivalent inducer eventually decreases ternary complex formation due to separate bivalent molecules independently binding each of the protein partners rather than bridging a ternary complex ${ }^{41}$.

Finally, we tested a third inducible system, this time exchanging ZA for tBID, a protein that does not bind KRas, but does interact with both Mcl1 and Bcl $\mathrm{XL}$. As expected, both the Raf-Bcl $\mathrm{XL}_{\mathrm{L}}$ and Raf-Mcl1 resulted in enhanced luciferase reporter gene expression (Figure 1D). We again observed a hook effect dependent on the expression of the bivalent inducer. With low

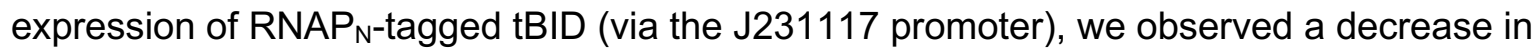
signal with increasing $\mathrm{Raf}-\mathrm{Bcl}_{\mathrm{XL}}$ inducer expression, although there was a significant signal over background even without IPTG induction. With the same low expression of the RNAP -tagged tBID, we observed an expected IPTG-dependent increase in RNAP activity with increasing RafMcl1 inducer expression. With high RNAP $_{\mathrm{N}}$-tagged tBID expression (driven by the J231114 promoter), we observed the same trends, though greatly magnified (Figure 1E). Previous work in our group and reported in the literature found that the binary PPI between Mcl1 and tBID is weaker than the PPI between $\mathrm{Bcl}_{\mathrm{xL}}$ and $\mathrm{BBID}^{82,85-86}$, which could explain these differences in signal response and varied hook effect magnitude.

Overall, these data indicated that the split RNAP system is capable of robustly detecting bivalent molecule-induced interactions between non-interacting protein pairs. Tuning bivalent inducers to properly drive a set of non-interacting partners without succumbing to the hook effect or losing potency is a major challenge in the rational design of inducible systems. Therefore, we next sought to develop a PACE system for optimizing PPI inducers, with the proof-of-concept goal being to overcome the hook effect.

\section{Design and validation of rePPI-G}

To convert our PPI inducer detection assay into a PACE system, we made two key changes. First, we cloned the ZB-Raf inducer from the IPTG inducible plasmid to the M13 bacteriophage's glll region under a high expression ProB promoter ${ }^{87}$ (Figure 2A). The high expression promoter should produce enough ZB-Raf inducer to decrease its overall activity via the "hook" effect. 
Second, we changed the downstream RNAP output protein from bacterial luciferase (LuxAB) to glll, the protein necessary for bacteriophage replication. We next assessed whether ZB-Raf phage can replicate in a manner dependent on the selectively induced interaction between ZA and KRas.

To measure phage replication, we used overnight growth assays. In this assay, a small number of phage $\left(10^{3} / \mathrm{mL}\right)$ are used to infect engineered $E$. Coli host cells for 24 hours, at which point the final phage titer is quantified using plaque assays ${ }^{88}$. We found that ZB-Raf phage that infected cells expressing RNAP $\mathrm{N}_{\mathrm{N}}$-tagged tBID and RNAP -tagged KRas did not replicate at all (Figure S2). However, when these phage infected cells expressing RNAP $\mathrm{N}_{\mathrm{N}}$ tagged $\mathrm{ZA}$ and RNAP -tagged KRas, they replicated $>30,000$-fold to a population of $\sim 10^{7} / \mathrm{mL}$ (Figure S2). These results confirm that the materials for the rePPI-G system can drive the replication of engineered phage based on their production of a PPI inducer. Next, we sought to transition from these overnight phage growth assays to a more stringent, continuously evolving PACE format.

We initially performed the PACE of ZB-Raf phage with two different host cells, each featuring a different selection system design. One set was transformed with the combination of a RNAP $\mathrm{N}^{-}$ tagged ZA plasmid and a RNAP -tagged $K R$ as plasmid, along with a mutagenesis plasmid to increase genetic variation (Figure 2A). The second set of cells were designed to provide phage with their missing glll protein regardless of inducer activity. To accomplish this, we first cloned a tetracycline-repressed phage shock promoter upstream of glll so that both tetracycline addition and phage infection are required for glll expression ${ }^{83}$. This selection pressure-free genetic "drift" plasmid was transformed into the second set of host cells, along with a mutagenesis plasmid and a plasmid encoding the third antibiotic resistance gene. We began the PACE experiment with an equal mixture of these two cell populations in two lagoons, with tetracycline added to one lagoon to activate the "drift" expression cassette. After 39h, the tetracycline-treated lagoon (i.e., active "drift") had 400-fold higher phage titers than the lagoon without tetracycline, confirming "drift" cell efficacy (Figure 2B). After 47h we removed the "drift" cell population, thus increasing the overall evolutionary selection pressure and allowing noninfectious phage variants to wash out of the "drift" lagoon. Between the $47 \mathrm{~h}$ and $63 \mathrm{~h}$ timepoints, the phage titer in the lagoon without active "drift" cells increased by $10,000 x$, while the active "drift" lagoon retained its high titers, indicating the presence of active phage in both lagoons. Next, another PACE experiment was conducted with ZB-Raf-expressing phage without "drift" cells and with the addition of a negative selection plasmid (Figure S3). This new plasmid expresses glllneg, a dominant negative mutant of glll, under the control of an orthogonal RNAP promoter controlled by an orthogonal RNAP ${ }_{C}$ mutant fused to ZBneg, a protein that does not interact with any other protein in the PACE system. The phage again evolved within 48-72 hours, but produced a somewhat diminished final phage titer due low expression of glllneg from small off-target 
interactions. We then subjected the ZB-Raf phage from the $63 \mathrm{~h}$ timepoint of the original PACE to another round of PACE and observed a similar timing for phage titer increase, but significantly higher final phage titers (Figure S3). Based on phage titers, these experiments indicated multiple successful PACE runs.

We next used overnight growth assays to assess whether the phage from these PACE experiments possessed different replication properties when compared to wild type phage. We first compared wildtype vs. evolved phage replication with different $\mathrm{RNAP}_{\mathrm{N}}$-tagged $\mathrm{ZA}$ expression levels. We found that the original ZB-Raf inducer phage replicated 21-fold less when a lower level (J23117 promoter) of RNAP ${ }_{\mathrm{N}}$-tagged ZA is expressed (Figure 2C). By contrast, the evolved phage replicated with identical efficiency regardless of the $R N A P_{N}$-tagged $Z A$ expression level. In addition, these evolved phage remained incapable of replicating with cells expressing RNAP ${ }_{N}$-tagged tBID and RNAP ${ }_{C}$-tagged KRas, confirming that these phage are

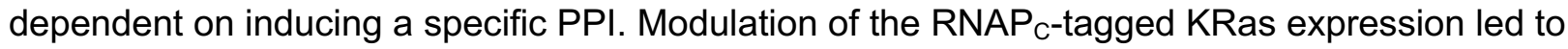
increased replication with increasing $\mathrm{RNAP}_{\mathrm{C}}$ expression for both the wildtype and evolved phage (Figure 2C). Notably, however, the evolved phage replicated over 300 -fold more overall than wildtype phage at both RNAPC expression levels. Taken together, these data confirm the PACE experiments generated evolved phage that replicate more effectively than wildtype phage while remaining dependent on selective PPI induction.

The observed PACE-evolved phage could have mutated in at least three ways to increase RNAP assembly at high ZB-Raf expression levels and thus mitigate the "hook" effect: 1) the inducer promoter strength could have been weakened to reduce ZB-Raf concentration; 2) the ZB affinity for ZA could have weakened to match the KRas-Raf interaction; or 3) the affinity of Raf for KRas could have increased to match the ZB-ZA interaction. To assess these possibilities, we Sanger-sequenced individual phage variants from each lagoon to determine which components evolved most frequently in the PACE experiments. We found that all variants had single or double mutations in the ZB region (Figure $3 A$ ) and only observed one mutation in the Raf protein $(\mathrm{N} 71 \mathrm{~K})$ that was rare after the first PACE, but common after the second negative selection PACE. No mutations were observed in the ProB promoter region. We cloned the most common variant, termed ZBF(3-2), into an IPTG-inducible expression vector and tested to what

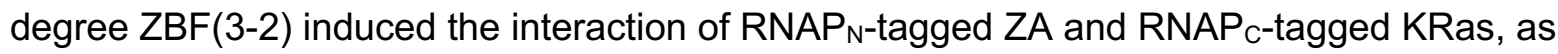
compared to ZB-Raf. ZBF(3-2) produced up to a 6-fold increase in RNAP activity compared to ZB-Raf at all inducer expression levels and did not decrease in activity with moderate inducer expression (10 $\mu \mathrm{M}$ IPTG), demonstrating increased resistance to the "hook" effect (Figure 3B). We next cloned each ZB or Raf variant as a fusion to the $\operatorname{RNAP}_{\mathrm{C}}$ or $\mathrm{RNAP}_{\mathrm{N}}$, respectively, to perform a binary PPI luciferase assay that measures variant binding to ZA or KRas respectively. RNAP $_{C}$-tagged ZB variants produced a 3- to 17-fold reduction RNAP activity when co- 
expressed with $\mathrm{RNAP}_{\mathrm{N}}$-tagged $\mathrm{ZA}$, indicating that the evolution selected solely for mutations that negatively impact ZB-ZA assembly and avoid the "hook" effect (Figure 3C). The Raf(N71K) mutation increased KRas-Raf assembly by $\sim 3-$ fold, again supporting the hypothesis that the ZBRaf inducer evolved to balance each PPI's relative affinity (Figure 3D). After this successful proof-of-concept evolution, we sought to expand our scope to a new PPI and evolve an inducer composed of short peptides.

\section{Expanding target scope of rePPI-G}

One challenge when identifying new protein targets for split reporter-based systems is avoiding false negatives stemming from either inherent instability in the new protein fusion or interference with split reporter assembly or activity. To avoid this pitfall, we first developed an assay to determine whether a new protein of interest (POI) can be fused to split RNAP reporters while retaining high signal-to-noise. We replaced the evolved $R_{N A P_{N}}$ with the wildtype $R N A P_{N}$, which is capable of reassembling with the RNAP R $_{C}$ with or without a PPI (Figure S4) ${ }^{74,89}$. We fused several POIs to either the RNAP or RNAP $_{C}$ and co-expressed these fusions with a cognate RNAP $_{C}$ or RNAP fusion protein capable of folding and reassembling to form an active RNAP. Using this assay, we rapidly identified GABARAP and LC3B as protein targets with high RNAP activity, albeit with >5-fold less activity than previously validated targets, KRas and tBID (Figure S5). Neither GABARAP nor LC3B greatly increased RNAP folding or expression, as evinced by their low background activity when fused to the evolved RNAPN (Figure S5 and S6).

We next searched the literature for peptides that bind GABARAP and/or LC3B. A recent publication screened and identified peptides from human proteins that selectively bind various autophagic adaptor proteins, including GABARAP and LC3B ${ }^{90}$. Two short peptides from the ULK1 and Fyco1 proteins possessed particularly high affinities for GABARAP and LC3B, respectively. We cloned $\mathrm{PPI}$ inducers composed of ZB or ZB(E6K, N23T), now termed eZB, fused to either ULK1 or Fyco1 and measured their ability to activate the split RNAP with GABARAP or LC3B fused to the RNAP . Both eZB-ULK1 and ZB-ULK1 induced RNAP activity when ZA and GABARAP were fused to the RNAP ${ }_{N}$ and RNAP $_{C}$, respectively (Figure 4A). ZBFyco1 induced a strong interaction between ZA-LC3B, and a weaker, yet significant, interaction between ZA-GABARAP (Figure 4B). Notably, increasing concentrations of the ZB-ULK1 and eZB-ULK1 inducers resulted in enhanced RNAP activity. Furthermore, the eZB-ULK1 inducer produced higher overall signal compared to ZB-ULK1, suggesting that the weaker eZB-ZA interaction is closer in affinity to the ULK1-GABARAP interaction than ZB-ZA. These data led to the hypothesis that the ULK1-GABARAP interaction is low affinity and thus a prime candidate for optimization via rePPI-G. 
To test this possibility, we cloned the ZB-ULK1 and eZB-ULK1 inducers into the bacteriophage genome and altered the RNAP output gene to glll. Host $E$. Coli cells were transformed with

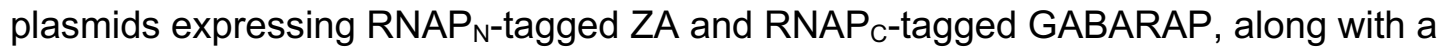
mutagenesis plasmid, and we performed a PACE experiment using these host cells and two lagoons, one containing ZB-ULK1 inducer phage and one containing eZB-ULK1 inducer phage. After 48h, eZB-ULK1 phage titers increased $\sim 50$-fold while ZB-ULK1 titers remained the same (Figure 4C). However, after $84 \mathrm{~h}$, both PPI inducer phage populations remained below $10^{7}$ phage $/ \mathrm{mL}$, indicating a stagnation in the evolution. Both repeating the evolution under the same conditions and increasing the evolutionary selection pressure by decreasing the expression of RNAP $_{\mathrm{N}}$-tagged ZA resulted in similarly low phage titers (Figure S7). We speculate that these evolutions were repeatedly stuck in activity minima that may require numerous mutations to overcome. While very rare phage with mutational combinations might escape the relative activity minima, it could take days or even weeks for these to overtake the population, especially given the rigorous selection pressure that continuous flow represents. Fine-tuning the PACE experiment components has proven successful in similar situations but can require weeks or months of optimization and evolution ${ }^{74}$. To sidestep this challenge, we developed an alternative evolution strategy.

\section{Rapidly overcoming local activity minima in rePPI-G}

Similar to an activity minimum, immeasurable starting activity is a common roadblock when carrying out PACE experiments. This challenge has typically been overcome by performing numerous rounds of phage assisted noncontinuous evolution/selection (PANCE/S) or traditional antibiotic selection screening ${ }^{91-93}$. Although these methods can take months to develop and achieve suitable activity to begin a PACE experiment, they are excellent at amplifying rare variants from a large pool. We hypothesized that a short round of "Phage-Assisted NonContinuous Selection" (PANCS), a combination of PANCE ${ }^{91}$ and PACS ${ }^{80}$, could quickly amplify rare PACE variants capable of escaping the PPI inducer activity minima.

Before performing the PANCS experiment, we first amplified the final phage titers from previous PACE experiments in a selection-free manner using 1059 cells, which supply glll in an activityindependent manner ${ }^{68}$. Next, we diluted these phage 10,000-fold into host $E$. Coli cells identical to those used in the PACE experiment, which express RNAP $_{\mathrm{N}}$-tagged $Z A$, RNAP $_{\mathrm{C}}$-tagged GABARAP, and glll when the RNAP is assembled. Importantly, we decided not to include a mutagenesis plasmid, thereby both decreasing the chance of genetic recombination and testing our hypothesis that highly mutated variants existed in the PACE. After the first round of PANCS the phage populations increased 630- and 158-fold (Figure 5A). We then performed another round of PANCS by diluting each sample to a concentration of $\sim 10^{5}$ phage/mL and incubating them overnight with host cells. Both phage populations increased 10,000-fold after the second 
round of PANCS, indicating the phage population had developed increased PPI induction efficacy. We then combined the PANCS amplified phage populations and subjected them to a final PACE experiment. After three days of PACE, phage achieved 20,000-fold greater titers than eZB-ULK1 inducer phage under continuous flow (Figure 5A). Moreover, the final phage population was more readily detectable using one of the most stringent assessments of phage activity, the activity dependent plaque assay. This experiment is similar to an overnight growth assay, except the phage and host cell mixture are incubated together in an agar gel matrix ${ }^{88}$. Only phage that acquire enough glll to replicate robustly can inhibit host cell growth to a degree that is detectable by eye. After the first PACE experiment plaques were noticeable, but not robust. After the PANCS and second PACE experiment plaques were very clearly visible (Fig $5 A)$.

\section{Characterization of evolved eZB-ULK1 variants}

Having successfully evolved the phage to replicate significantly more than wildtype, we Sanger sequenced the phage from each PACE experiment to assess the mutations in each binding partner. The first three PACE experiments resulted in three unique dominant phage. The mutant from the first PACE, ZU(1), had a deletion at base pair 1081 that changed the ending ULK1 coding sequence from -PAQFPGDLVA* to the remarkably similar, yet more positively charged PRNFRGT* (Fig 5B). The mutant from the second PACE experiment, which included an increase in selection pressure after $48 \mathrm{~h}$, produced a phage, $\mathrm{ZU}\left(1^{*}\right)$ with a deletion at base pair 1084 that resulted in the identical amino acid sequence. The replicate of the original PACE experiment did not produce any deletion mutants, instead resulting in a double mutant in the ULK1 sequence, ZU(2). Phage resulting from the PANCS and final PACE experiment, ZU(3), contained the ULK1 base pair 1081 deletion along with a threonine-to-alanine mutation within the amino acid sequence after the base pair deletion $\left(T^{\star} 21 \mathrm{~A}\right)$. Furthermore, this variant had three mutations in the eZB domain (A1T, Q18R, W21R). These evolved inducers were cloned into the IPTG-inducible plasmids used previously and assessed for improved induction of the ZA and GABARAP PPI. Both ZU(1) and ZU(2) had 6- to 10-fold increases in RNAP activity at all concentrations of inducer, as compared with wildtype phage (Figure 5C). Strikingly, these mutants also had nearly identical activity profiles at all IPTG concentrations. These mutants also exhibited no increase in RNAP activity between moderate and high inducer expression at lower RNAP $_{\mathrm{N}}$-tagged ZA expression levels (Figure S8), indicating a measurable "hook" effect. On the other hand, ZU(3) possessed 40- to 95-fold increased activity compared to the wildtype PPI inducer, a >2000-fold overall signal to noise ratio at maximum expression, and no "hook" effect, even with low RNAP $_{\mathrm{N}}$-tagged ZA expression (Figs. 5C and S8). Importantly, none of the variants dramatically increased off-target ZA-LC3B PPI induction, meaning the mutations did not impact overall split RNAP assembly and activity. After confirming the evolution was successful, 
we sought to characterize how these mutations impact the individual PPIs in our engineered and evolved bifunctional molecular glues.

We cloned each unique ULK1 variant to the RNAP to $_{\mathrm{N}}$ assess changes in the ULK1/GABARAP interaction. Both the ULK1(bp1081del) and ULK1(T354A, A370E) mutants had a 20-fold increase in RNAP activity compared to ULK1(wt) when paired with GABARAP (Figure 5D). Interestingly, the ULK1(T354A, A370E) mutant also had a small, yet significant, 2-fold increase in LC3B binding. Somewhat surprisingly, the ZU(3) ULK1 mutant had a 2-fold decrease in GABARAP binding compared to the ZU(1) and ZU(2) ULK1 variants. To determine how the $\mathrm{ZU}(3)$ inducer evolved to radically improve overall inducer activity, we cloned its eZB mutant as a fusion to the RNAP . We observed no difference in RNAP activity between eZB(A1T, Q18R, $W 21 R$ ) and eZB when interacting with $Z A$, but we did observe a small, significant increase in RNAP activity when ZB(A1T, Q18R, W21R) interacted with the off-target tBID (Figure S9). Binary binding assays with ULK1 variants and LC3B confirmed that these mutations also conferred a small increase in binding affinity to these off-target proteins (Figure S10). We next cloned eZB and eZB(A1T, Q18R, W21R) into IPTG inducible plasmids to assess whether this new promiscuous peptide was capable of inducing a PPI between either ZA and LC3B or ZA and GABARAP. We observed a small, yet significant, increase in RNAP signal when the eZB(A1T, Q18R, W21R) peptide was expressed at its highest concentration and inducing the interaction between ZA and LC3B (Figure S11). It is possible that these nearly undetectable interactions reflect the molecular glue's ability to bring multiple proteins within close proximity to one another.

Finally, we validated that changes in PPI affinities observed and optimized in E. Coli translate to mammalian cell experiments. To measure induced PPIs in mammalian cells, we inserted the highest affinity ULK1 mutant - ULK1(bp1081del) - within a Myc-tagged protein, and then performed a co-immunoprecipitation to probe for its interaction partners, GABARAPL1-1/2. Expression of the ULK1(bp1081del) mutant construct pulled down significantly more endogenous GABARAP protein compared to the ULK1(wt) sequence (Figure 5E). Expression of the Myc-tagged protein alone resulted in no GABARAP signal, confirming this interaction is dependent on the ULK1 peptide. Experiment replication and image quantification confirmed this observation is consistent and significant (Figure S12). 


\section{Discussion}

During our validation of rePPI-G, we discovered affinity-altering mutations that parallel those found in previous studies. For example, our group used PACS to perform deep mutational scanning on the KRas/Raf1 interaction interface ${ }^{80}$. While most mutations in Raf1 resulted in either no change or a decrease in enrichment, N71K resulted in the most positive enrichment score, indicating this mutation increases the interaction affinity. Not only did we also observe the N71K mutation in this work when evolving the ZB-Raf1 inducer, but we also confirmed it increases the interaction affinity between KRas and Raf1 $\sim 3$-fold (Figure 3). Crystal structures of the PPI complex reveal that the Raf1 N71 residue is $\sim 2.5 \AA$ away from the negatively charged D33 residue on KRas, an optimal distance for a potential salt bridge interaction ${ }^{81}$. The mutations discovered in our eZB-ULK1 evolution also matched those found in previous studies by other groups. In-depth point mutation screens of the ULK1 LC3 interaction region (LIR), the same peptide used in our evolution, revealed T47 as a key residue for LC3B binding ${ }^{90}$. This observation mirrored the T47A, A63E double mutant discovered in our evolution that demonstrated a small yet significant increase in LC3B binding when compared to the wildtype ULK1 peptide (Figure 5D). These data, along with the mammalian cell co-immunoprecipitation experiment and previous work ${ }^{74,80,94}$, support the theory that observed changes in activity translate beyond the split RNAP system to biologically relevant interactions.

While designing and validating rePPI-G we also learned several key lessons in bivalent inducer engineering. The "hook" effect makes fine-tuning any PPI inducer a challenge, particularly in a therapeutic context, where target protein concentrations are dynamic and uncontrollable. We consistently found that alterations in protein binder affinity were sufficient to overcome the "hook" effect. When inducing a tBID/KRas interaction, for instance, the higher affinity Bcl $\mathrm{xL}_{\mathrm{xL}} \mathrm{tBID}$ interaction results in a dramatic "hook" effect relative to the Mcl1/tBID interaction (Figure 1D). In the case of the ZB-Raf inducer evolution, all ZB mutations resulted in weaker ZA binding. This not only diminished the "hook" effect, but also produced more ZA/KRas interaction at all expression levels, implying changes that reduce the "hook" effect may actually produce an overall more efficient PPI inducer (Figure 3B and 4C). Finally, in the case of the eZB-ULK1 inducer, we found that increasing the potency of one ligand (ULK1) provided increased potency until a "hook" effect developed, at which point the paired protein binder (eZB) became the limiting factor in increasing inducer potency (Figure 5). These results make it clear that increased interaction affinity of one protein binder does not always result in overall increased molecular glue potency. Together, these findings suggest a new strategy for discovering bivalent PPI inducers, including PROTACs, wherein binders with different affinities for protein "A" and protein "B" are screened as a matrix of unique bivalent molecules to discover the optimal molecular glue for each target interaction. 
In this proof-of-concept work, engineered bifunctional molecular glues - which already have affinity for both targets - were our starting point, but rePPI-G could in principle use naïve libraries to evolve molecules de novo. While theoretically feasible, implementing phage libraries in PACE often presents technical challenges, including glll recombination or limited library size, that preclude identification of functional starting points. Nonetheless, based on our results here and in previous PACE-based evolutionary campaigns ${ }^{91-93}$, a combination of PANCE/PACS with PACE may be the solution to implementing libraries with rePPI-G. An advantage of the rePPI-G pipeline is that the same split RNAP-based biosensors used for the evolution can also be used to measure target activity in live mammalian cells ${ }^{85-86}$. Most recently, we showcased a PPI evolution system for PACE that features both positive and negative selection, which permitted the rapid reprogramming of PPI specificity via $\mathrm{PACE}^{94}$, further demonstrating the potential of PACE-based evolutions and suggesting the rePPI-G platform can be expanded to evolutions of selective molecular glues.

This work showcases evolution as a strategy to find unique and even unimaginable solutions to biological challenges. In the case of eZB-ULK1, rePPI-G discovered single base pair deletions that dramatically increased individual PPI affinity and a triad of mutations that conferred a subtle promiscuity resulting in optimized molecular glue activity. More broadly, now that we have validated the proximity dependent split RNAP as a system to select for and evolve molecular glues, this opens up the possibility of implementing this selection scheme with other evolution technologies. For example, implementing the molecular glue selection with the yeast-based Orthorep, mammalian-based mPACE, or VEGAS systems would allow for evolution of molecules that drive the interactions between proteins that cannot be expressed in E. coli, a key limitation of PACE-based methods ${ }^{70-73}$. As the repertoire of rapid evolutionary technologies expands, so too will our ability to harness evolution to solve problems in synthetic biology and therapeutic design. 


\section{Methods}

\section{DNA Plasmid Cloning}

All plasmids were cloned using Phusion or Q5 DNA polymerases (NEB) for PCR amplification of specific DNA fragments, Gibson Assembly to generate new plasmids from PCR fragments, $\mathrm{DH} 10 \beta$ E. Coli cells for transformation, and Sanger Sequencing via the University of Chicago Comprehensive Cancer Center DNA Sequencing and Genotyping Facility to verify plasmid sequence fidelity and integrity ${ }^{95}$. DNA primers were ordered from Integrated DNA Technologies (IDT) or Sigma-Aldrich. All plasmid maps, descriptions, annotations, and gene DNA/amino acid sequences are available in supplementary materials online along with links to full DNA sequences for each plasmid used in this study. Additionally, key vectors will be deposited to Addgene for distribution and all vectors are available upon request.

\section{Split RNAP luciferase assay in $E$. Coli}

Chemically competent S1030 or S2060 E. Coli cells (available from Addgene) were transformed with $\mathrm{RNAP}_{\mathrm{N}}$ and $\mathrm{RNAP}_{\mathrm{C}}$ fusion protein plasmids that also contain $\mathrm{T7}$ driven LuxAB. If a third plasmid is needed, as is the case when testing a PPI inducer, chemically competent cells of the doubly transformed $E$. Coli were prepared and transformed with the third plasmid. E. Coli colonies were then picked in quadruplicate into $500 \mu \mathrm{L}$ of LB (miller) broth (US Biological Life Sciences) with antibiotics (GoldBio) in a 96-well deep well plate and incubated in a shaker at 220 RPM for $12-16$ h overnight. $10 \mu \mathrm{L}$ of each overnight growth was then transferred to $500 \mu \mathrm{L}$ of LB media containing antibiotics and any inducing small molecule such as IPTG (GoldBio). After 4-5h when the cells are between 0.3 and $0.5 \mathrm{OD}_{600}, 150 \mu \mathrm{L}$ of cells were transferred to a 96-well imaging plate (costar) and assayed for luminescence and $\mathrm{OD}_{600}$ on a Synergy $\mathrm{NeO} 2$ hybrid multimode plate reader (BioTek Instruments, Inc.).

\section{Quantifying Phage Titer with Plaque Assays}

Chemically competent S1030 or S2060 E. Coli cells (available from Addgene) were transformed with plasmid 6-64 that contains phage shock promoter driven glll. These cells are also termed 1059 cells here and in the literature. A colony of 6-64 containing cells was picked into antibiotics media and grown to $0.5-0.9 \mathrm{OD}_{600}$, or grown overnight and diluted to $0.5-0.9 \mathrm{OD}_{600} .50 \mu \mathrm{L}$ of diluted 6-64 cells were then aliquoted into $41.5 \mu \mathrm{L}$ Eppendorf tubes for each phage sample to be measured. $1 \mu \mathrm{L}$ of phage sample was pipetted to the first tube, mixed vigorously by flicking, and then $1 \mu \mathrm{L}$ of that solution was transferred to the next tube using a new tip to prevent inflated titers. This serial dilution was repeated for the remaining tubes so that the final tube represents a $125,000,000 x$ dilution from the original phage sample. $750 \mu \mathrm{L}$ of $50{ }^{\circ} \mathrm{C}$ top agar $(7 \mathrm{~g} / \mathrm{L}$ agar, $25 \mathrm{~g} / \mathrm{L}$ LB broth) was added to each tube and then quickly transferred in order of increasing phage concentration to a $10 \mathrm{~cm}$ quad plate coated in bottom agar $(15 \mathrm{~g} / \mathrm{L}$ agar, $25 \mathrm{~g} / \mathrm{L} \mathrm{LB}$ broth). Plaques should be visible after $12-24 \mathrm{~h}$ and can be counted to quantify phage titer. Note: 
place warm top agar in a tight-fitting Styrofoam container whenever pipetting at room temperature to delay agar solidification.

\section{Quantifying Phage Activity with Overnight Growth Assays}

To quantitatively compare phage activity between samples, we first measured each sample's phage titers as described above. Next we diluted each phage sample in LB broth containing appropriate antibiotics to the same titer (final concentration between 1000 and 10,000 phage/mL for best signal to noise). $500 \mu \mathrm{L}$ of the diluted phage were added to a 96 deep well plate in triplicate. A colony of S1030 or S2060 E. Coli cells containing the desired activity selection plasmids (for example tagged split RNAP biosensors) was picked into each well and the plate was incubated for $24 \mathrm{~h}$. The contents were spun down and immediately used in a plaque assay to quantify final phage titers as described above.

\section{Phage Assisted Continuous Evolutions}

For the most in depth and up to date protocol for performing a phage assisted continuous evolution (PACE) see the following reference ${ }^{69}$. One significant difference in all of our PACE protocols is that we use a more affordable Forma 3960 environmental chamber (ThermoFisher Scientific) rather than a warm room to house and conduct PACE experiments ${ }^{74}$. To perform rePPI-G specifically, we first transformed S1030 E. Coli cells with an MP4 mutagenesis plasmid (available from Addgene), an $\mathrm{RNAP}_{\mathrm{N}}$-tagged protein expression vector, and an $\mathrm{RNAP}_{\mathrm{C}}$-tagged protein expression vector that also contains CGG driven glll expression. These cells were plated on LB agar containing appropriate antibiotics along with $20 \mathrm{mM}$ glucose to suppress the MP4 mutagenesis plasmid activity. The next day, a single colony was picked for each chemostat into two $15 \mathrm{~mL}$ culture tubes with $6 \mathrm{~mL}$ of LB media, and one tube containing $20 \mathrm{mM}$ glucose and the other containing $20 \mathrm{mM}$ arabinose. Davis Rich Media (also called Harvard Custom Media C, US Biological Life Sciences) was prepared or purchased and autoclaved in 7.5L batches. Davis Rich Media supplement (also called Harvard Custom Media A, US Biological Life Sciences) was prepared or purchased and sterile filtered as previously described in the references above. The media base was added to the environmental chamber to equilibrate to 37 degrees Celsius overnight. The next day the sterile filtered supplement was added to the media base by a flame to prevent contamination. After 14-16h of growth, the arabinose induced cells were checked for greatly diminished growth compared to the glucose repressed cells, which suggests an active MP4 plasmid. The glucose-containing tube was added to a $500 \mathrm{~mL}$ chemostat and $100-200 \mathrm{~mL}$ of Davis Rich Media at 37 degrees Celsius was pumped into the chemostat over the course of two hours. Once cells reached approximately 0.4-0.8 OD the lagoon pump was activated to allow chemostat cells to fill the $50 \mathrm{~mL}$ lagoons to a volume of $20 \mathrm{~mL}$ at a flow rate of $1 \mathrm{v} / \mathrm{h}$. Syringes containing $20 \% \mathrm{w} / \mathrm{v}$ arabinose and $18 \mathrm{mM}$ $\mathrm{MgSO}_{4}$, which we find improves phage titers by $\sim 5$ fold, in water began pumping into each 
lagoon at a rate of $0.5 \mathrm{~mL} / \mathrm{h}$. To activate "drift" cells, $2 \mu \mathrm{g} / \mathrm{mL}$ of tetracycline (GoldBio) was added to the syringe solution along with the previously mentioned arabinose and $\mathrm{MgSO}_{4}$. The PACE system was allowed to equilibrate concentration for 1-2h before adding $1 \mathrm{~mL}$ of the desired phage at a concentration of of $>10^{9}$ phage $/ \mathrm{mL}$ to each lagoon, at which point the lagoon and syringe pumps were paused for 30-60 minutes to provide phage ample time for initial host cell infection. After 30-60 minutes, the syringe and lagoon pumps were reactivated. Samples were extracted from lagoons in the morning and evening, and plaque assays performed each day to monitor phage titer. $1 \mu \mathrm{L}$ of phage sample was then directly added to a PCR to amplify phage variants for Sanger sequencing. New chemostats were added after $48 \mathrm{~h}$ to ensure cells remained at maximal infectivity. Also, all chemostats and lagoons were kept below $50 \%$ maximum volume and constantly stirred via magnetic stir bars to ensure cells were properly aerated.

\section{Phage Assisted Noncontinuous Selection}

For the most detailed and up to date protocol on PANCE experiments see the following reference $^{69}$. PANCS experiments were carried out as follows. $50 \mu \mathrm{L}$ of phage samples from PACE experiments were added to $5 \mathrm{~mL}$ of LB broth along with a colony of 1059 cells. After $24 \mathrm{~h}$ of growth the sample was spun down, and the supernatant was passed through a $0.2 \mu \mathrm{M}$ filter to remove all $E$. Coli cells while collecting phage. Phage titer was measured as described above. Phage samples were diluted $10,000 x$ into $500 \mu \mathrm{L}$ LB broth along with $1 \%$ by volume of overnight selection cell culture. Samples were grown at 37 degrees Celsius for $20-24 \mathrm{~h}$, spun down, and then phage titer was quantified. The resulting phage were passed through a $0.2 \mu \mathrm{m}$ filter to remove all $E$. Coli cells and then diluted to a final phage titer of $10^{5}$ phage $/ \mathrm{mL}$ in $500 \mu \mathrm{L}$ LB broth along with $1 \%$ by volume of overnight selection cell culture. As with PACE samples, 1 $\mu \mathrm{L}$ of phage sample was then directly added to a PCR to amplify phage variant DNA for Sanger sequencing. This cycle can be repeated with variable phage dilution or selection cells to alter selection pressure until desired activity emerges indicating a successful selection or phage titer drops below $10^{3}$ phage/mL indicating no active phage were present in the original sample.

\section{Mammalian Cell Culture}

HEK293T cells (ATCC) were maintained in DMEM (high glucose, L-glutamine, phenol red, sodium pyruvate; obtained from Gibco or Hyclone) supplemented with $10 \%$ fetal bovine serum (FBS, Gibco/Life Technologies, Qualified US origin) and 1\% penicillin/streptomycin (P/S, Gibco/Life Technologies). Multiple biological replicates were performed with cells from different passages below 20 and freshly thawed aliquots. Transfections were carried out using Lipofectamine 3000 (Thermo Fisher). 


\section{Co-Immunoprecipitation of Myc-tagged Proteins}

For co-immunoprecipitation, cells were lysed in SDS-free RIPA ( $0 \mathrm{mM}$ Tris, $150 \mathrm{mM} \mathrm{NaCl}, 0.5 \%$ deoxycholate, $0.1 \%$ SDS, $1.0 \%$ Triton $\mathrm{X}-100$, $\mathrm{pH} 7.4$ ) supplemented with PMSF and a protease inhibitor cocktail on ice for 10 minutes, followed by centrifugation at 13,000xg for 20 min. Protein (20 $\mathrm{\mu g}$, "input") was removed and lysate was added to Protein G Dynabeads (Invitrogen: 10003D) pre-incubated with c-Myc antibody (Santa Cruz sc-40). After 12 hours of enrichment, beads were washed (3x, SDS-free RIPA) and total protein eluted with $50 \mathrm{mM}$ glycine, "output." Both input and output protein were subjected to separation by SDS-PAGE. After SDS-PAGE, proteins were transferred onto methanol-preactivated Immobilon-P PVDF membranes (pore size $0.45 \mu \mathrm{m}$; Millipore) using a semi-dry transfer cell. After transfer, membranes were treated in accordance with standard Western blotting procedures, using a solution of $3 \% \mathrm{BSA}$ (ThermoFisher) in either TBST (20 mM Tris, $\mathrm{pH} 7.5,150 \mathrm{mM} \mathrm{NaCl}, 0.1 \%$ Tween-20). Membranes were probed with GABARAP (Abcam), c-Myc (Santa Cruz), and calnexin (Abcam) antibodies overnight. Following secondary antibody incubation, membranes were visualized using SuperSignal West Pico PLUS chemiluminescent substrate (ThermoFisher) and recorded on a chemiluminescent Western blot imaging system (Azure Biosystems C300). 


\section{Associated Content}

The Supporting Information is available free of charge at XXX.

Additional detailed experimental information, extended data (Figures S1- S12), and plasmid lists.

\section{Author Contributions}

B.C.D. and J.D. conceived of and planned experiments. J.D. cloned the vectors for this study with assistance from V.L., and conducted luciferase assays, evolutions and selections, and data analyses. S.A. conducted co-immunoprecipitations and Western blot analyses. J.D. and B.C.D. wrote the paper with input from all coauthors.

\section{Notes}

B.C.D. has filed a patent on the proximity-dependent split RNAP technology and is also a founder and holds equity in Tornado Bio, Inc.

\section{Acknowledgements}

The authors thank Dr. S. Ahmadiantehrani for assistance with preparing this manuscript.

This work by the National Cancer Institute (R21 CA21775) and the National Institute of General Medical Sciences (R35 GM119840) of the National Institutes of Health. J.D. was supported by a Multi-disciplinary Training program in Cancer Research from the National Institutes of Health (T32 CA009594). 
A

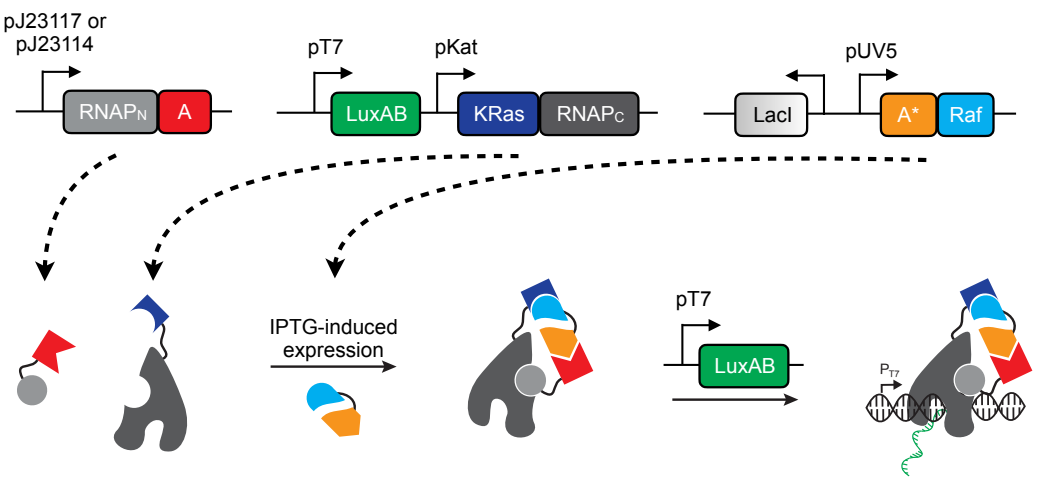

B

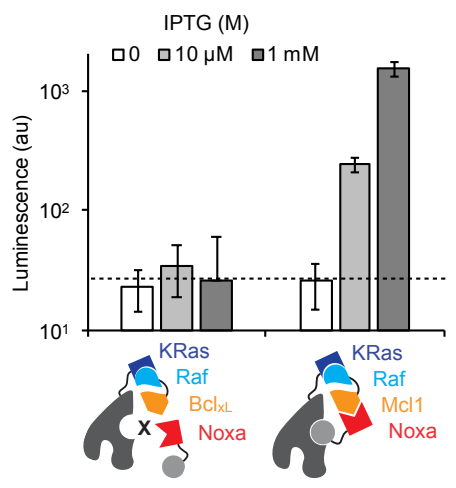

C

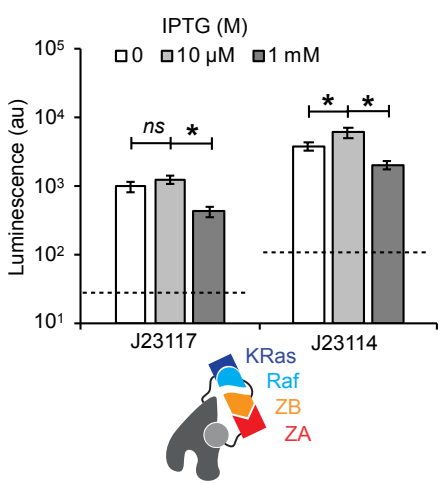

D

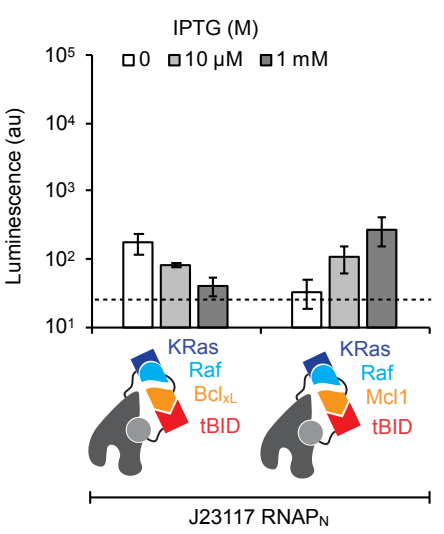

E

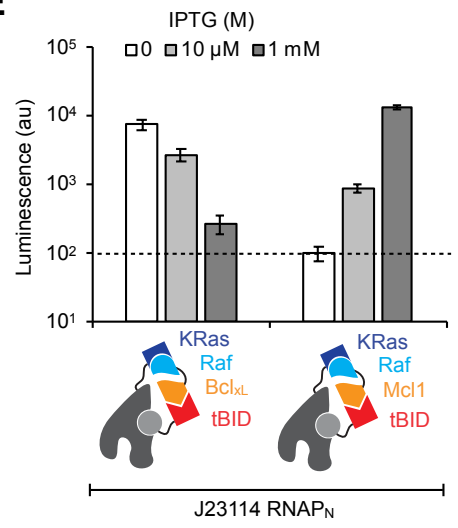

Figure 1. Split RNAP biosensors detect molecular glue-induced PPIs. (A) Schematic of plasmid maps used in the inducer detection assay and cartoon representation of inducer detection assay mechanism. (B) Luminescence data from molecular glues with a mismatched PPI (Bcl $\left.\right|_{x L}$ and Noxa) and an interacting PPI (Mcl1 and Noxa) at various molecular glue expression levels as controlled by IPTG concentration. (C) Luminescence data obtained with the ZB-Raf molecular

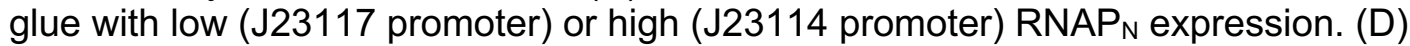
Luminescence data from $\mathrm{Bcl}_{\mathrm{xL}}$-Raf and Mcl1-Raf molecular glues with low RNAPN $\mathrm{R}_{\mathrm{N}}$ expression. (E) Luminescence data from $\mathrm{Bcl}_{\mathrm{xL}}$-Raf and Mcl1-Raf molecular glues with high $\mathrm{RNAP}_{\mathrm{N}}$ expression. Dashed lines indicate baseline luminescence signal from PPI-independent RNAP reassembly as reported in Figure $\mathrm{S} 1 .^{*}=p<0.05, n s=p>0.05$ 


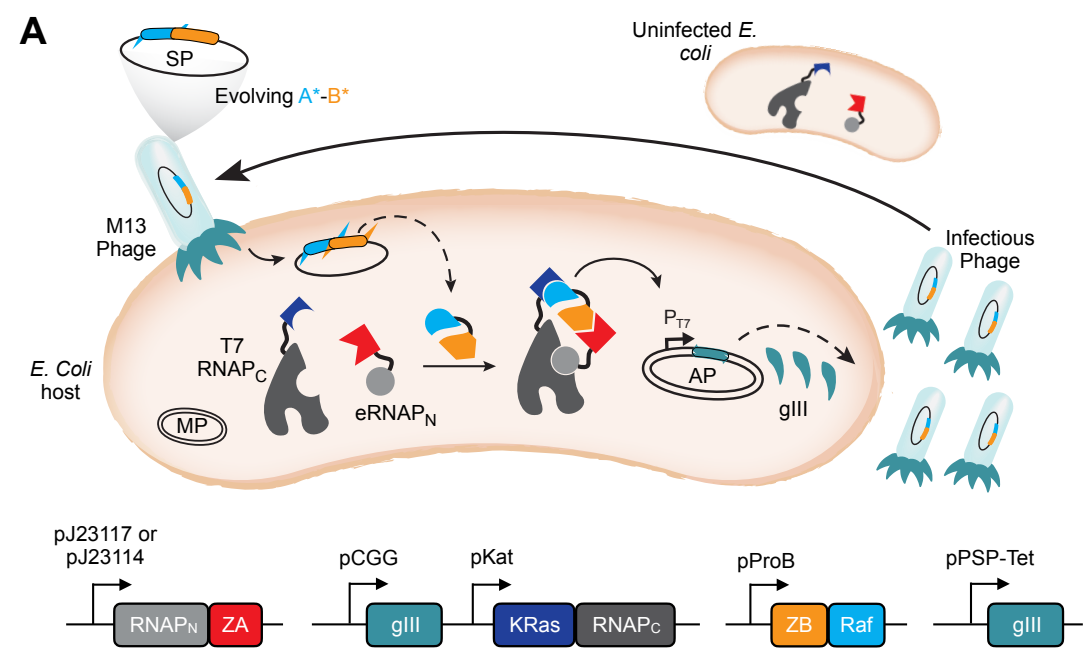

B

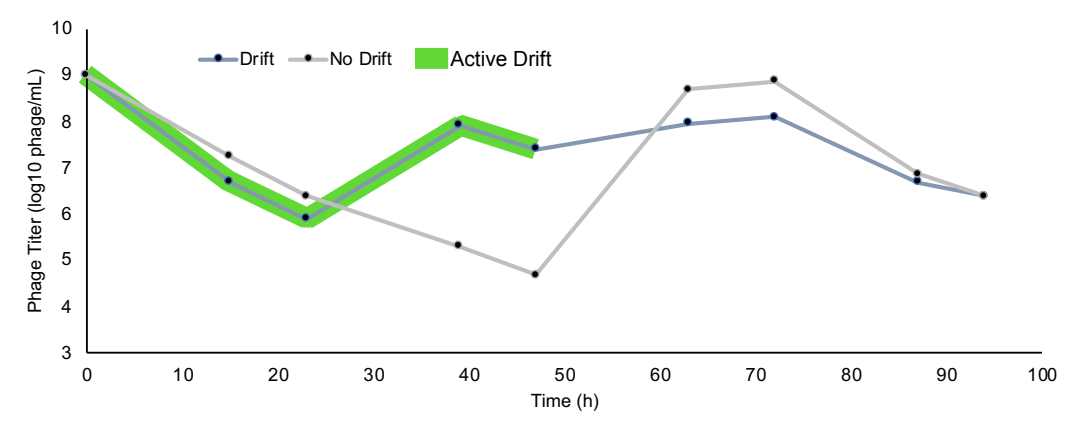

C
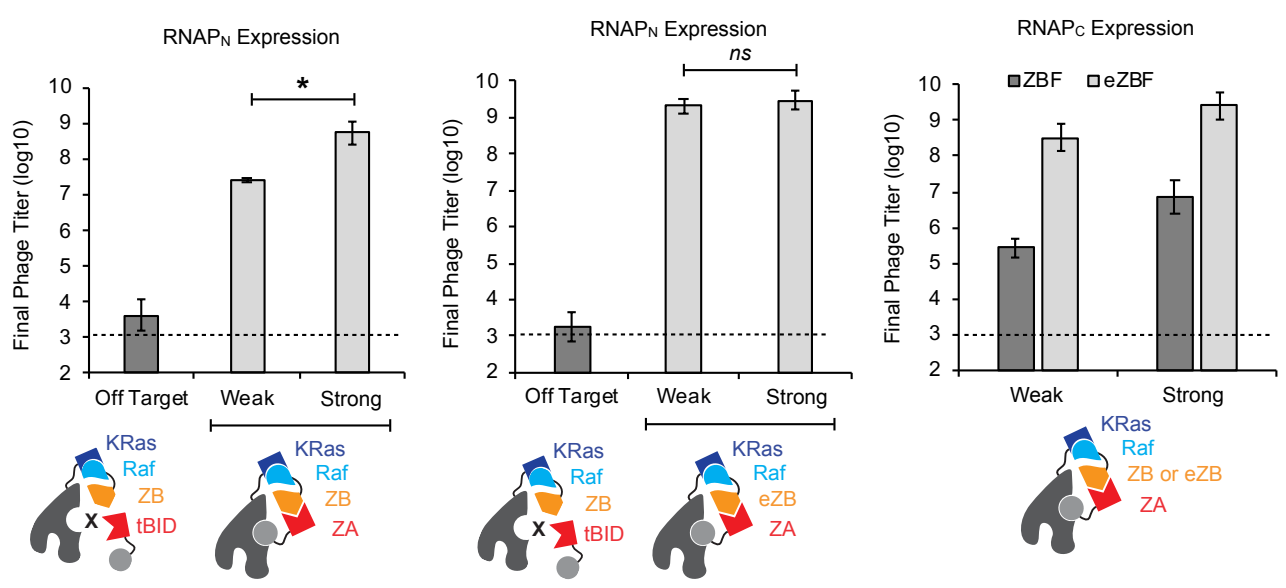

Figure 2. Rapid evolution of protein-protein interaction molecular glues (rePPI-G) to overcome the "hook" effect. (A) Schematic of plasmid maps used in the molecular glue PACE and cartoon representation of molecular glue PACE components and mechanism. (B) ZB-Raf molecular glue phage titers over time during PACE experiment. Green background indicates active genetic "drift" cells. (C) Quantification of phage replication in overnight growth assays. Wildtype ZB-Raf molecular glue phage activity with a mismatched target (tBID and ZB) and a weak (J23117 promoter) or strong (J23114 promoter) RNAP $_{\mathrm{N}}$-tagged ZA (left panel) compared to the PACEevolved ZB-Raf molecular glue phage with the same targets (center panel). Wildtype (dark) compared to evolved (light) ZB-Raf molecular glues with weak (sd6 RBS) or strong (SD8 RBS) RNAP $_{c}$-tagged KRas expression. ${ }^{*}=p<0.05, n s=p>0.05$ 
A

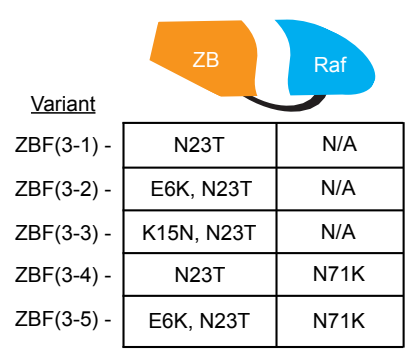

C

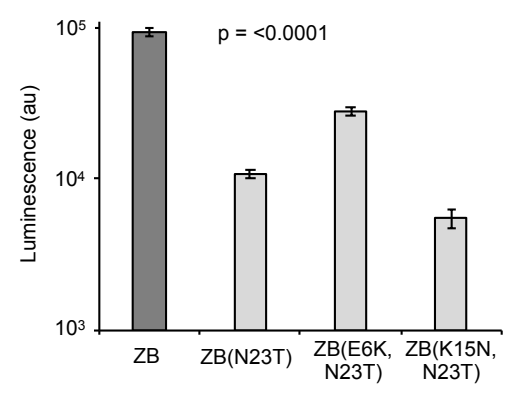

$O_{0}^{Z B}$
B

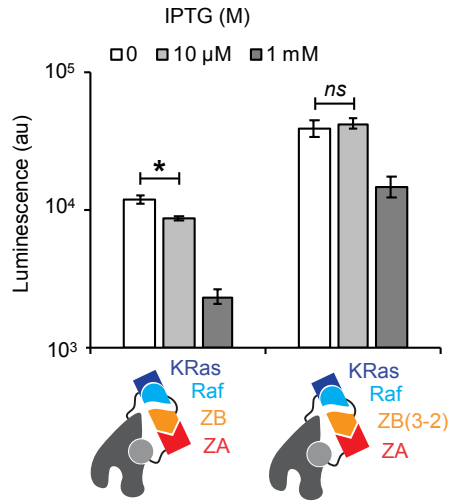

D

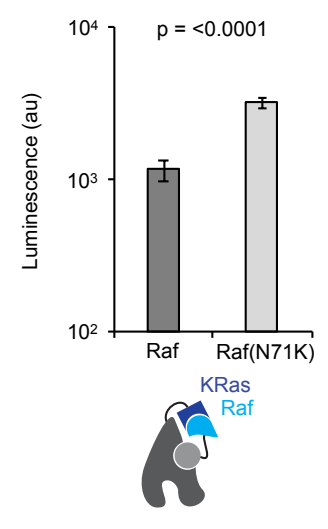

Figure 3. rePPI-G improved molecular glue activity by altering binary interaction affinities. (A) Common mutations discovered in the PACE experiment. (B) Luminescence data of ZB-Raf or ZBF(3-2) molecular glues inducing an interaction between ZA and KRas at different concentrations. $(C)$ Luminescence data of individual binary interactions between RNAP $\mathrm{C}_{\text {-tagged }}$ $\mathrm{ZB}$ or ZB variants with RNAP ${ }_{\mathrm{N}}$-tagged $\mathrm{ZA}$. All mutants had $\mathrm{p}=<0.0001$ compared to $\mathrm{ZB}$ (wt)

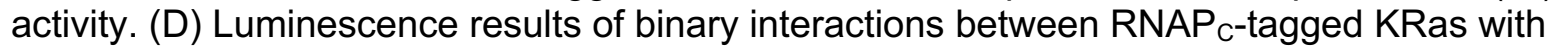
RNAP $_{N}$-tagged Raf or $\operatorname{Raf}(N 71 K) . p=<0.0001 .{ }^{*}=p<0.05, n s=p>0.05$. 
A

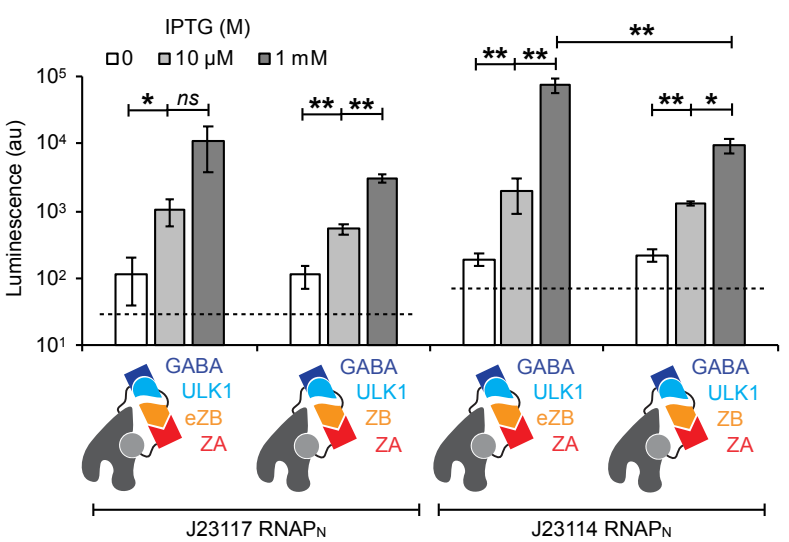

B

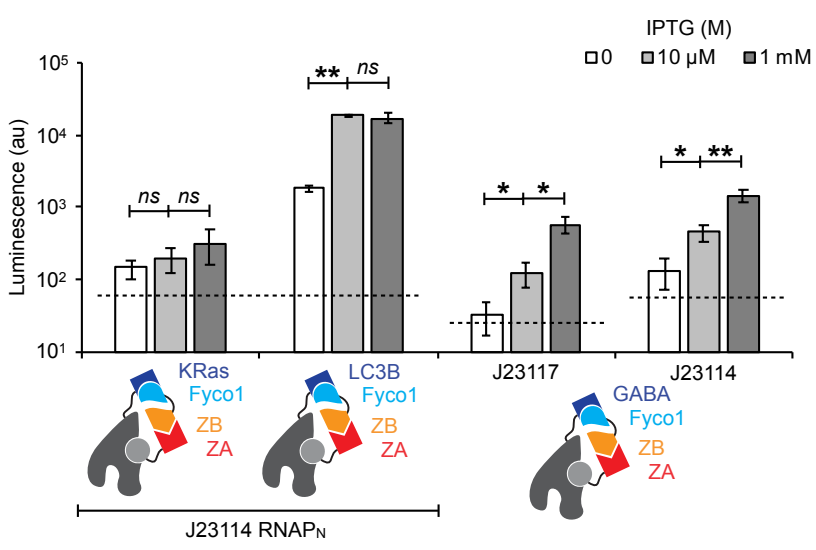

C

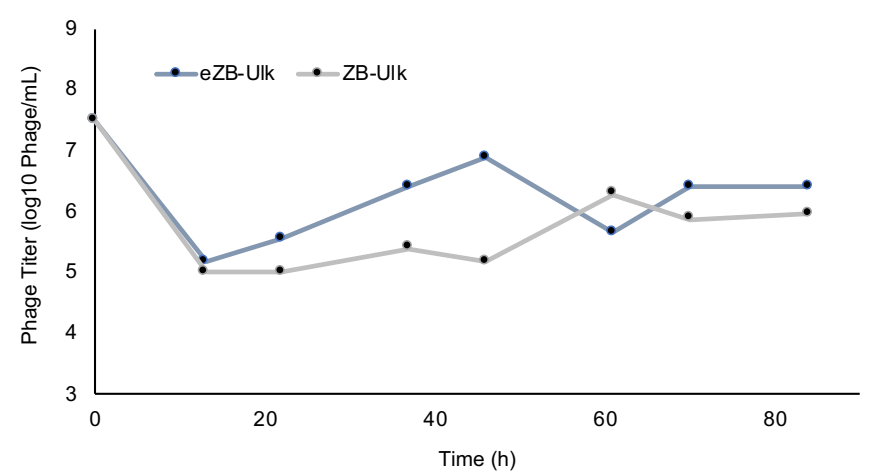

Figure 4. Expanding target scope of rePPI-G. (A) Luminescence data from ZB-ULK1 and eZBULK1 molecular glues inducing the interaction between GABARAP and ZA at low (J23117 promoter) and high (J23114 promoter) RNAP $_{N}$ expression levels. (B) Luminescence data obtained with the ZB-Fyco1 molecular glue with an off-target PPI (KRas and ZA) and two ontarget PPIs (LC3B or GABARAP and ZA). (C) PACE of eZB-ULK1 (blue) and ZB-ULK1 (gray). Dashed lines represent background RNAP assembly as reported in Figure S6. ${ }^{*}=p<0.05,{ }^{* *}=$ $p<0.005, n s=p>0.05$ 
A

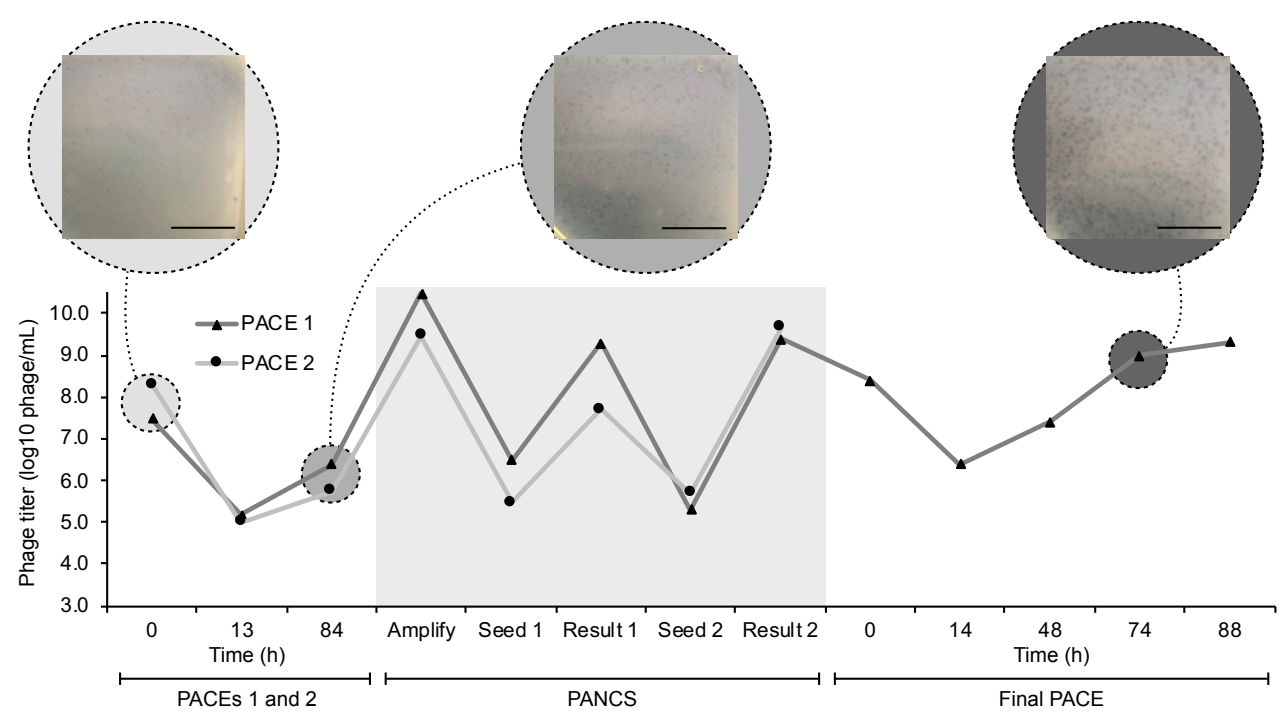

B

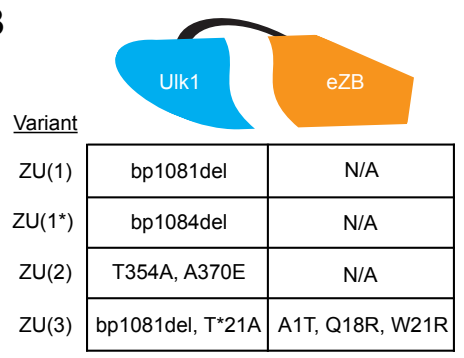

C

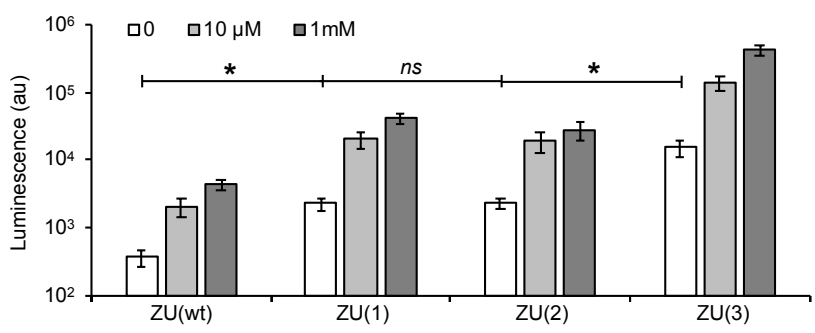

D

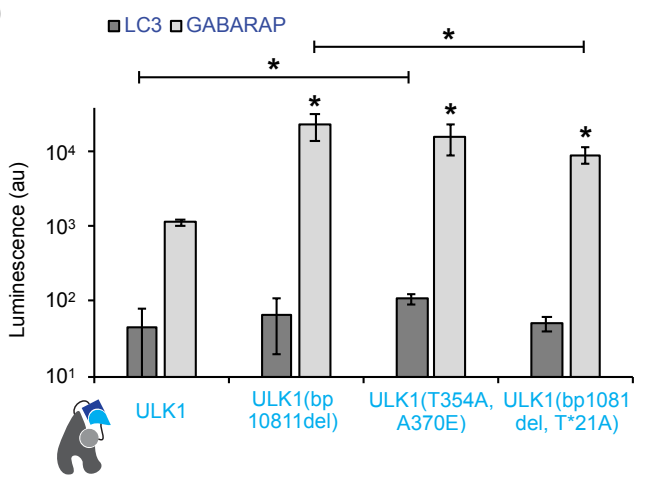

E

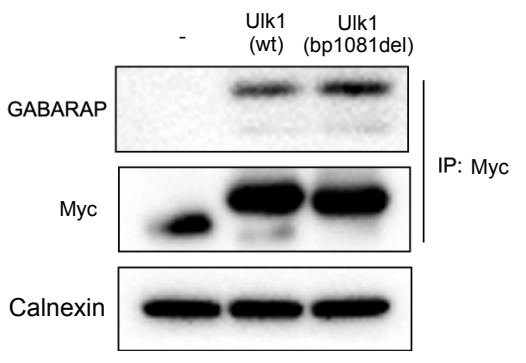

Figure 5. Combination of continuous and noncontinuous phage assays escape local activity minima in rePPI-G. (A) Phage titers across full eZB-ULK1 molecular glue evolution including original PACE, PACE replicate, PANCS (light background), and final PACE along with pictures of activity dependent plaque assay results at different time points (scale bar $=1 \mathrm{~cm})$. (B) Common mutations discovered from rePPI-G eZB-ULK1 evolutions. (C) Luminescence data measuring each IPTG inducible eZB-ULK1 variant's ability to induce the interaction between

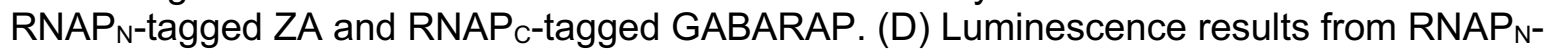
tagged ULK1 rePPI-G variants paired with either RNAPc-tagged LC3B (dark) or GABARAP 
(light). (E) Co-immunoprecipitation of Myc-tagged ULK1 or ULK1(bp1081del) to measure GABARAP pulldown efficiency with calnexin loading control and Myc output control. 


\section{References}

1. Kim, S. J.; Fernandez-Martinez, J.; Nudelman, I.; Shi, Y.; Zhang, W.; Raveh, B.; Herricks, T.; Slaughter, B. D.; Hogan, J. A.; Upla, P.; Chemmama, I. E.; Pellarin, R.; Echeverria, I.; Shivaraju, M.; Chaudhury, A. S.; Wang, J.; Williams, R.; Unruh, J. R.; Greenberg, C. H.; Jacobs, E. Y.; Yu, Z.; de la Cruz, M. J.; Mironska, R.; Stokes, D. L.; Aitchison, J. D.; Jarrold, M. F.; Gerton, J. L.; Ludtke, S. J.; Akey, C. W.; Chait, B. T.; Sali, A.; Rout, M. P., Integrative structure and functional anatomy of a nuclear pore complex. Nature 2018, 555 (7697), 475-482.

2. Perkins, J. R.; Diboun, I.; Dessailly, B. H.; Lees, J. G.; Orengo, C., Transient protein-protein interactions: structural, functional, and network properties. Structure 2010, 18 (10), 1233-43.

3. Jaremko, M. J.; Davis, T. D.; Corpuz, J. C.; Burkart, M. D., Type II non-ribosomal peptide synthetase proteins: structure, mechanism, and protein-protein interactions. Nat Prod Rep 2020, 37 (3), 355-379.

4. Pawson, T.; Scott, J. D., Signaling through scaffold, anchoring, and adaptor proteins. Science 1997, 278 (5346), 2075-80.

5. Li, J.; Mahajan, A.; Tsai, M. D., Ankyrin repeat: a unique motif mediating protein-protein interactions. Biochemistry 2006, 45 (51), 15168-78.

6. Zeytuni, N.; Zarivach, R., Structural and functional discussion of the tetra-trico-peptide repeat, a protein interaction module. Structure 2012, 20 (3), 397-405.

7. Cheng, F.; Zhao, J.; Wang, Y.; Lu, W.; Liu, Z.; Zhou, Y.; Martin, W. R.; Wang, R.; Huang, J.; Hao, T.; Yue, H.; Ma, J.; Hou, Y.; Castrillon, J. A.; Fang, J.; Lathia, J. D.; Keri, R. A.; Lightstone, F. C.; Antman, E. M.; Rabadan, R.; Hill, D. E.; Eng, C.; Vidal, M.; Loscalzo, J., Comprehensive characterization of protein-protein interactions perturbed by disease mutations. Nat Genet 2021, 53 (3), 342-353.

8. Pardoll, D. M., The blockade of immune checkpoints in cancer immunotherapy. Nat Rev Cancer 2012, 12 (4), 252-64.

9. Dang, C. V., MYC on the path to cancer. Cell 2012, 149 (1), 22-35.

10. McCubrey, J. A.; Steelman, L. S.; Chappell, W. H.; Abrams, S. L.; Wong, E. W.; Chang, F.; Lehmann, B.; Terrian, D. M.; Milella, M.; Tafuri, A.; Stivala, F.; Libra, M.; Basecke, J.; Evangelisti, C.; Martelli, A. M.; Franklin, R. A., Roles of the Raf/MEK/ERK pathway in cell growth, malignant transformation and drug resistance. Biochim Biophys Acta 2007, 1773 (8), 1263-84.

11. Caughey, B.; Lansbury, P. T., Protofibrils, pores, fibrils, and neurodegeneration: separating the responsible protein aggregates from the innocent bystanders. Annu Rev Neurosci 2003, 26, 267-98.

12. Chen, Y.; Dorn, G. W., 2nd, PINK1-phosphorylated mitofusin 2 is a Parkin receptor for culling damaged mitochondria. Science 2013, 340 (6131), 471-5.

13. Cuadrado, A.; Rojo, A. I.; Wells, G.; Hayes, J. D.; Cousin, S. P.; Rumsey, W. L.; Attucks, O. C.; Franklin, S.; Levonen, A. L.; Kensler, T. W.; Dinkova-Kostova, A. T., Therapeutic targeting of the NRF2 and KEAP1 partnership in chronic diseases. Nat Rev Drug Discov 2019, 18 (4), 295-317.

14. Kaminska, B., MAPK signalling pathways as molecular targets for anti-inflammatory therapy--from molecular mechanisms to therapeutic benefits. Biochim Biophys Acta 2005, 1754 (1-2), 253-62.

15. Wynn, T. A., Cellular and molecular mechanisms of fibrosis. J Pathol 2008, 214 (2), 199-210. 
16. Bonacci, W.; Teng, P. K.; Afonso, B.; Niederholtmeyer, H.; Grob, P.; Silver, P. A.; Savage, D. F., Modularity of a carbon-fixing protein organelle. Proc Natl Acad Sci U S A 2012, 109 (2), 478-83.

17. Divine, R.; Dang, H. V.; Ueda, G.; Fallas, J. A.; Vulovic, I.; Sheffler, W.; Saini, S.; Zhao, Y. T.; Raj, I. X.; Morawski, P. A.; Jennewein, M. F.; Homad, L. J.; Wan, Y. H.; Tooley, M. R.; Seeger, F.; Etemadi, A.; Fahning, M. L.; Lazarovits, J.; Roederer, A.; Walls, A. C.; Stewart, L.; Mazloomi, M.; King, N. P.; Campbell, D. J.; McGuire, A. T.; Stamatatos, L.; Ruohola-Baker, H.; Mathieu, J.; Veesler, D.; Baker, D., Designed proteins assemble antibodies into modular nanocages. bioRxiv 2020.

18. Lajoie, M. J.; Boyken, S. E.; Salter, A. I.; Bruffey, J.; Rajan, A.; Langan, R. A.; Olshefsky, A.; Muhunthan, V.; Bick, M. J.; Gewe, M.; Quijano-Rubio, A.; Johnson, J.; Lenz, G.; Nguyen, A.; Pun, S.; Correnti, C. E.; Riddell, S. R.; Baker, D., Designed protein logic to target cells with precise combinations of surface antigens. Science 2020, 369 (6511), 1637-1643.

19. Chen, Z.; Kibler, R. D.; Hunt, A.; Busch, F.; Pearl, J.; Jia, M.; VanAernum, Z. L.; Wicky, B. I. M.; Dods, G.; Liao, H.; Wilken, M. S.; Ciarlo, C.; Green, S.; El-Samad, H.; Stamatoyannopoulos, J.; Wysocki, V. H.; Jewett, M. C.; Boyken, S. E.; Baker, D., De novo design of protein logic gates. Science 2020, 368 (6486), 78-84.

20. Nitta, T.; Wang, Y.; Du, Z.; Morishima, K.; Hiratsuka, Y., A printable active network actuator built from an engineered biomolecular motor. Nat Mater 2021.

21. Choe, J. H.; Watchmaker, P. B.; Simic, M. S.; Gilbert, R. D.; Li, A. W.; Krasnow, N. A.; Downey, K. M.; Yu, W.; Carrera, D. A.; Celli, A.; Cho, J.; Briones, J. D.; Duecker, J. M.; Goretsky, Y. E.; Dannenfelser, R.; Cardarelli, L.; Troyanskaya, O.; Sidhu, S. S.; Roybal, K. T.; Okada, H.; Lim, W. A., SynNotch-CAR T cells overcome challenges of specificity, heterogeneity, and persistence in treating glioblastoma. Sci Transl Med 2021, 13 (591).

22. Yoshikawa, M.; Yoshii, T.; Ikuta, M.; Tsukiji, S., Synthetic Protein Condensates That Inducibly Recruit and Release Protein Activity in Living Cells. J Am Chem Soc 2021, 143 (17), 6434-6446.

23. Scott, D. E.; Bayly, A. R.; Abell, C.; Skidmore, J., Small molecules, big targets: drug discovery faces the protein-protein interaction challenge. Nat Rev Drug Discov 2016, 15 (8), 533-50.

24. Arkin, M. R.; Wells, J. A., Small-molecule inhibitors of protein-protein interactions: progressing towards the dream. Nat Rev Drug Discov 2004, 3 (4), 301-17.

25. Arkin, M. R.; Tang, Y.; Wells, J. A., Small-molecule inhibitors of protein-protein interactions: progressing toward the reality. Chem Biol 2014, 21 (9), 1102-14.

26. Mabonga, L.; Kappo, A. P., Protein-protein interaction modulators: advances, successes and remaining challenges. Biophys Rev 2019, 11 (4), 559-581.

27. Lu, H.; Zhou, Q.; He, J.; Jiang, Z.; Peng, C.; Tong, R.; Shi, J., Recent advances in the development of protein-protein interactions modulators: mechanisms and clinical trials. Signal Transduct Target Ther 2020, 5 (1), 213.

28. Souers, A. J.; Leverson, J. D.; Boghaert, E. R.; Ackler, S. L.; Catron, N. D.; Chen, J.; Dayton, B. D.; Ding, H.; Enschede, S. H.; Fairbrother, W. J.; Huang, D. C.; Hymowitz, S. G.; Jin, S.; Khaw, S. L.; Kovar, P. J.; Lam, L. T.; Lee, J.; Maecker, H. L.; Marsh, K. C.; Mason, K. D.; Mitten, M. J.; Nimmer, P. M.; Oleksijew, A.; Park, C. H.; Park, C. M.; Phillips, D. C.; Roberts, A. W.; Sampath, D.; Seymour, J. F.; Smith, M. L.; Sullivan, G. M.; Tahir, S. K.; Tse, C.; Wendt, M. D.; Xiao, Y.; Xue, J. C.; Zhang, H.; Humerickhouse, R. A.; Rosenberg, S. H.; Elmore, S. W., ABT-199, a potent and selective BCL-2 inhibitor, achieves antitumor activity while sparing platelets. Nat Med 2013, 19 (2), 202-8. 
29. Gerry, C. J.; Schreiber, S. L., Unifying principles of bifunctional, proximity-inducing small molecules. Nat Chem Biol 2020, 16 (4), 369-378.

30. Maniaci, C.; Ciulli, A., Bifunctional chemical probes inducing protein-protein interactions. Curr Opin Chem Biol 2019, 52, 145-156.

31. Zarzycka, B.; Kuenemann, M. A.; Miteva, M. A.; Nicolaes, G. A. F.; Vriend, G.; Sperandio, O., Stabilization of protein-protein interaction complexes through small molecules. Drug Discov Today 2016, 21 (1), 48-57.

32. Andrei, S. A.; Sijbesma, E.; Hann, M.; Davis, J.; O'Mahony, G.; Perry, M. W. D.; Karawajczyk, A.; Eickhoff, J.; Brunsveld, L.; Doveston, R. G.; Milroy, L. G.; Ottmann, C., Stabilization of protein-protein interactions in drug discovery. Expert Opin Drug Discov 2017, 12 (9), 925-940.

33. Schneekloth, A. R.; Pucheault, M.; Tae, H. S.; Crews, C. M., Targeted intracellular protein degradation induced by a small molecule: En route to chemical proteomics. Bioorg Med Chem Lett 2008, 18 (22), 5904-8.

34. Lu, J.; Qian, Y.; Altieri, M.; Dong, H.; Wang, J.; Raina, K.; Hines, J.; Winkler, J. D.; Crew, A. P.; Coleman, K.; Crews, C. M., Hijacking the E3 Ubiquitin Ligase Cereblon to Efficiently Target BRD4. Chem Biol 2015, 22 (6), 755-63.

35. Lai, A. C.; Toure, M.; Hellerschmied, D.; Salami, J.; Jaime-Figueroa, S.; Ko, E.; Hines, J.; Crews, C. M., Modular PROTAC Design for the Degradation of Oncogenic BCR-ABL. Angew Chem Int Ed Engl 2016, 55 (2), 807-10.

36. Schapira, M.; Calabrese, M. F.; Bullock, A. N.; Crews, C. M., Targeted protein degradation: expanding the toolbox. Nat Rev Drug Discov 2019, 18 (12), 949-963.

37. Paiva, S. L.; Crews, C. M., Targeted protein degradation: elements of PROTAC design. Curr Opin Chem Biol 2019, 50, 111-119.

38. Mayor-Ruiz, C.; Bauer, S.; Brand, M.; Kozicka, Z.; Siklos, M.; Imrichova, H.; Kaltheuner, I. H.; Hahn, E.; Seiler, K.; Koren, A.; Petzold, G.; Fellner, M.; Bock, C.; Muller, A. C.; Zuber, J.; Geyer, M.; Thoma, N. H.; Kubicek, S.; Winter, G. E., Rational discovery of molecular glue degraders via scalable chemical profiling. Nat Chem Biol 2020, 16 (11), 1199-1207.

39. An, S.; Fu, L., Small-molecule PROTACs: An emerging and promising approach for the development of targeted therapy drugs. EBioMedicine 2018, 36, 553-562.

40. Gao, H.; Sun, X.; Rao, Y., PROTAC Technology: Opportunities and Challenges. ACS Med Chem Lett 2020, 11 (3), 237-240.

41. Miles, L. E., Properties, variants, and applications of the immunoradiometric assay method. Ric Clin Lab 1975, 5 (1), 59-72.

42. Ray, S. S.; Nowak, R. J.; Brown, R. H., Jr.; Lansbury, P. T., Jr., Small-molecule-mediated stabilization of familial amyotrophic lateral sclerosis-linked superoxide dismutase mutants against unfolding and aggregation. Proc Natl Acad Sci U S A 2005, 102 (10), 3639-44.

43. Tan, Z.; Wortman, M.; Dillehay, K. L.; Seibel, W. L.; Evelyn, C. R.; Smith, S. J.; Malkas, L. H.; Zheng, Y.; Lu, S.; Dong, Z., Small-molecule targeting of proliferating cell nuclear antigen chromatin association inhibits tumor cell growth. Mol Pharmacol 2012, 81 (6), 811-9. 
44. Nemetski, S. M.; Cardozo, T. J.; Bosch, G.; Weltzer, R.; O'Malley, K.; Ejigiri, I.; Kumar, K. A.; Buscaglia, C. A.; Nussenzweig, V.; Sinnis, P.; Levitskaya, J.; Bosch, J., Inhibition by stabilization: targeting the Plasmodium falciparum aldolase-TRAP complex. Malar J 2015, 14, 324.

45. Sijbesma, E.; Visser, E.; Plitzko, K.; Thiel, P.; Milroy, L. G.; Kaiser, M.; Brunsveld, L.; Ottmann, C., Structure-based evolution of a promiscuous inhibitor to a selective stabilizer of protein-protein interactions. Nat Commun 2020, 11 (1), 3954.

46. Ferrara, M.; Sessa, G.; Fiore, M.; Bernard, F.; Asteriti, I. A.; Cundari, E.; Colotti, G.; Ferla, S.; Desideri, M.; Buglioni, S.; Trisciuoglio, D.; Del Bufalo, D.; Brancale, A.; Degrassi, F., Small molecules targeted to the microtubule-Hec1 interaction inhibit cancer cell growth through microtubule stabilization. Oncogene 2018, 37 (2), 231-240.

47. Li, Z.; Wang, C.; Wang, Z.; Zhu, C.; Li, J.; Sha, T.; Ma, L.; Gao, C.; Yang, Y.; Sun, Y.; Wang, J.; Sun, X.; Lu, C.; Difiglia, M.; Mei, Y.; Ding, C.; Luo, S.; Dang, Y.; Ding, Y.; Fei, Y.; Lu, B., Allele-selective lowering of mutant HTT protein by HTT-LC3 linker compounds. Nature 2019, 575 (7781), 203-209.

48. Tang, C.; Mo, X.; Niu, Q.; Wahafu, A.; Yang, X.; Qui, M.; Ivanov, A. A.; Du, Y.; Fu, H., Hypomorph mutation-directed small-molecule protein-protein interaction inducers to restore mutant SMAD4suppressed TGF-beta signaling. Cell Chem Biol 2021, 28 (5), 636-647 e5.

49. Hartman, A. M.; Elgaher, W. A. M.; Hertrich, N.; Andrei, S. A.; Ottmann, C.; Hirsch, A. K. H., Discovery of Small-Molecule Stabilizers of 14-3-3 Protein-Protein Interactions via Dynamic Combinatorial Chemistry. ACS Med Chem Lett 2020, 11 (5), 1041-1046.

50. Sijbesma, E.; Somsen, B. A.; Miley, G. P.; Leijten-van de Gevel, I. A.; Brunsveld, L.; Arkin, M. R.; Ottmann, C., Fluorescence Anisotropy-Based Tethering for Discovery of Protein-Protein Interaction Stabilizers. ACS Chem Biol 2020, 15 (12), 3143-3148.

51. Guillory, X.; Wolter, M.; Leysen, S.; Neves, J. F.; Kuusk, A.; Genet, S.; Somsen, B.; Morrow, J. K.; Rivers, E.; van Beek, L.; Patel, J.; Goodnow, R.; Schoenherr, H.; Fuller, N.; Cao, Q.; Doveston, R. G.; Brunsveld, L.; Arkin, M. R.; Castaldi, P.; Boyd, H.; Landrieu, I.; Chen, H.; Ottmann, C., Fragmentbased Differential Targeting of PPI Stabilizer Interfaces. J Med Chem 2020, 63 (13), 6694-6707.

52. Wolter, M.; Valenti, D.; Cossar, P. J.; Levy, L. M.; Hristeva, S.; Genski, T.; Hoffmann, T.; Brunsveld, L.; Tzalis, D.; Ottmann, C., Fragment-Based Stabilizers of Protein-Protein Interactions through ImineBased Tethering. Angew Chem Int Ed Engl 2020, 59 (48), 21520-21524.

53. Newman, M. J.; Benani, D. J., A review of blinatumomab, a novel immunotherapy. J Oncol Pharm Pract 2016, 22 (4), 639-45.

54. Linke, R.; Klein, A.; Seimetz, D., Catumaxomab: clinical development and future directions. MAbs 2010, 2 (2), 129-36.

55. Sedykh, S. E.; Prinz, V. V.; Buneva, V. N.; Nevinsky, G. A., Bispecific antibodies: design, therapy, perspectives. Drug Des Devel Ther 2018, 12, 195-208.

56. Labrijn, A. F.; Janmaat, M. L.; Reichert, J. M.; Parren, P., Bispecific antibodies: a mechanistic review of the pipeline. Nat Rev Drug Discov 2019, 18 (8), 585-608.

57. Arnold, F. H., Directed Evolution: Bringing New Chemistry to Life. Angew Chem Int Ed Engl 2018, 57 (16), 4143-4148. 
58. Bornscheuer, U. T.; Hauer, B.; Jaeger, K. E.; Schwaneberg, U., Directed Evolution Empowered Redesign of Natural Proteins for the Sustainable Production of Chemicals and Pharmaceuticals. Angew Chem Int Ed Engl 2019, 58 (1), 36-40.

59. Liang, A. D.; Serrano-Plana, J.; Peterson, R. L.; Ward, T. R., Artificial Metalloenzymes Based on the Biotin-Streptavidin Technology: Enzymatic Cascades and Directed Evolution. Acc Chem Res 2019, $52(3), 585-595$.

60. Davis, A. M.; Plowright, A. T.; Valeur, E., Directing evolution: the next revolution in drug discovery? Nat Rev Drug Discov 2017, 16 (10), 681-698.

61. Kuchner, O.; Arnold, F. H., Directed evolution of enzyme catalysts. Trends Biotechnol 1997, 15 (12), 523-30.

62. Brandenberg, O. F.; Chen, K.; Arnold, F. H., Directed Evolution of a Cytochrome P450 Carbene Transferase for Selective Functionalization of Cyclic Compounds. J Am Chem Soc 2019, 141 (22), 8989-8995.

63. Waldo, G. S., Genetic screens and directed evolution for protein solubility. Curr Opin Chem Biol 2003, $7(1), 33-8$.

64. Roodveldt, C.; Aharoni, A.; Tawfik, D. S., Directed evolution of proteins for heterologous expression and stability. Curr Opin Struct Biol 2005, 15 (1), 50-6.

65. Clackson, T.; Hoogenboom, H. R.; Griffiths, A. D.; Winter, G., Making antibody fragments using phage display libraries. Nature 1991, 352 (6336), 624-8.

66. Roberts, R. W.; Szostak, J. W., RNA-peptide fusions for the in vitro selection of peptides and proteins. Proc Natl Acad Sci U S A 1997, 94 (23), 12297-302.

67. Hanes, J.; Pluckthun, A., In vitro selection and evolution of functional proteins by using ribosome display. Proc Natl Acad Sci U S A 1997, 94 (10), 4937-42.

68. Esvelt, K. M.; Carlson, J. C.; Liu, D. R., A system for the continuous directed evolution of biomolecules. Nature 2011, 472 (7344), 499-503.

69. Miller, S. M.; Wang, T.; Liu, D. R., Phage-assisted continuous and non-continuous evolution. Nat Protoc 2020, 15 (12), 4101-4127.

70. Rix, G.; Watkins-Dulaney, E. J.; Almhjell, P. J.; Boville, C. E.; Arnold, F. H.; Liu, C. C., Scalable continuous evolution for the generation of diverse enzyme variants encompassing promiscuous activities. Nat Commun 2020, 11 (1), 5644.

71. Berman, C. M.; Papa, L. J., 3rd; Hendel, S. J.; Moore, C. L.; Suen, P. H.; Weickhardt, A. F.; Doan, N. D.; Kumar, C. M.; Uil, T. G.; Butty, V. L.; Hoeben, R. C.; Shoulders, M. D., An Adaptable Platform for Directed Evolution in Human Cells. J Am Chem Soc 2018, 140 (51), 18093-18103.

72. Hendel, S. J.; Shoulders, M. D., Directed evolution in mammalian cells. Nat Methods 2021, 18 (4), 346-357.

73. English, J. G.; Olsen, R. H. J.; Lansu, K.; Patel, M.; White, K.; Cockrell, A. S.; Singh, D.; Strachan, R. T.; Wacker, D.; Roth, B. L., VEGAS as a Platform for Facile Directed Evolution in Mammalian Cells.

Cell 2019, 178 (3), 748-761 e17. 
74. Pu, J.; Zinkus-Boltz, J.; Dickinson, B. C., Evolution of a split RNA polymerase as a versatile biosensor platform. Nat Chem Biol 2017, 13 (4), 432-438.

75. Pu, J.; Disare, M.; Dickinson, B. C., Evolution of C-Terminal Modification Tolerance in Full-Length and Split T7 RNA Polymerase Biosensors. Chembiochem 2019, 20 (12), 1547-1553.

76. Segall-Shapiro, T. H.; Meyer, A. J.; Ellington, A. D.; Sontag, E. D.; Voigt, C. A., A 'resource allocator' for transcription based on a highly fragmented T7 RNA polymerase. Mol Syst Biol 2014, 10, 742.

77. Choi, J.; Chen, J.; Schreiber, S. L.; Clardy, J., Structure of the FKBP12-rapamycin complex interacting with the binding domain of human FRAP. Science 1996, 273 (5272), 239-42.

78. Yin, P.; Fan, H.; Hao, Q.; Yuan, X.; Wu, D.; Pang, Y.; Yan, C.; Li, W.; Wang, J.; Yan, N., Structural insights into the mechanism of abscisic acid signaling by PYL proteins. Nat Struct Mol Biol 2009, 16 (12), 1230-6.

79. Pu, J.; Kentala, K.; Dickinson, B. C., Multidimensional Control of Cas9 by Evolved RNA PolymeraseBased Biosensors. ACS Chem Biol 2018, 13 (2), 431-437.

80. Zinkus-Boltz, J.; DeValk, C.; Dickinson, B. C., A Phage-Assisted Continuous Selection Approach for Deep Mutational Scanning of Protein-Protein Interactions. ACS Chem Biol 2019, 14 (12), 2757-2767.

81. Tran, T. H.; Chan, A. H.; Young, L. C.; Bindu, L.; Neale, C.; Messing, S.; Dharmaiah, S.; Taylor, T.; Denson, J. P.; Esposito, D.; Nissley, D. V.; Stephen, A. G.; McCormick, F.; Simanshu, D. K., KRAS interaction with RAF1 RAS-binding domain and cysteine-rich domain provides insights into RASmediated RAF activation. Nat Commun 2021, 12 (1), 1176.

82. Chen, L.; Willis, S. N.; Wei, A.; Smith, B. J.; Fletcher, J. I.; Hinds, M. G.; Colman, P. M.; Day, C. L.; Adams, J. M.; Huang, D. C., Differential targeting of prosurvival Bcl-2 proteins by their BH3-only ligands allows complementary apoptotic function. Mol Cell 2005, 17 (3), 393-403.

83. Carlson, J. C.; Badran, A. H.; Guggiana-Nilo, D. A.; Liu, D. R., Negative selection and stringency modulation in phage-assisted continuous evolution. Nat Chem Biol 2014, 10 (3), 216-22.

84. Ghosh, I.; Hamilton, A. D.; Regan, L., Antiparallel Leucine Zipper-Directed Protein Reassembly: Application to the Green Fluorescent Protein. Journal of the American Chemical Society 2000, 122 (23), 5658-5659.

85. Dewey, J. A.; Dickinson, B. C., Split T7 RNA polymerase biosensors to study multiprotein interaction dynamics. Methods Enzymol 2020, 641, 413-432.

86. Pu, J.; Dewey, J. A.; Hadji, A.; LaBelle, J. L.; Dickinson, B. C., RNA Polymerase Tags To Monitor Multidimensional Protein-Protein Interactions Reveal Pharmacological Engagement of Bcl-2 Proteins. J Am Chem Soc 2017, 139 (34), 11964-11972.

87. Rogers, J. K.; Guzman, C. D.; Taylor, N. D.; Raman, S.; Anderson, K.; Church, G. M., Synthetic biosensors for precise gene control and real-time monitoring of metabolites. Nucleic Acids Res 2015, 43 (15), 7648-60.

88. Dickinson, B. C.; Packer, M. S.; Badran, A. H.; Liu, D. R., A system for the continuous directed evolution of proteases rapidly reveals drug-resistance mutations. Nat Commun 2014, 5, 5352.

89. Wang, T.; Badran, A. H.; Huang, T. P.; Liu, D. R., Continuous directed evolution of proteins with improved soluble expression. Nat Chem Biol 2018, 14 (10), 972-980. 
90. Wirth, M.; Zhang, W.; Razi, M.; Nyoni, L.; Joshi, D.; O'Reilly, N.; Johansen, T.; Tooze, S. A.; Mouilleron, S., Molecular determinants regulating selective binding of autophagy adapters and receptors to ATG8 proteins. Nat Commun 2019, 10 (1), 2055.

91. Roth, T. B.; Woolston, B. M.; Stephanopoulos, G.; Liu, D. R., Phage-Assisted Evolution of Bacillus methanolicus Methanol Dehydrogenase 2. ACS Synth Biol 2019, 8 (4), 796-806.

92. Blum, T. R.; Liu, H.; Packer, M. S.; Xiong, X.; Lee, P. G.; Zhang, S.; Richter, M.; Minasov, G.; Satchell, K. J. F.; Dong, M.; Liu, D. R., Phage-assisted evolution of botulinum neurotoxin proteases with reprogrammed specificity. Science 2021, 371 (6531), 803-810.

93. Richter, M. F.; Zhao, K. T.; Eton, E.; Lapinaite, A.; Newby, G. A.; Thuronyi, B. W.; Wilson, C.; Koblan, L. W.; Zeng, J.; Bauer, D. E.; Doudna, J. A.; Liu, D. R., Phage-assisted evolution of an adenine base editor with improved Cas domain compatibility and activity. Nat Biotechnol 2020, 38 (7), 883-891.

94. Xie, V. C.; Pu, J.; Metzger, B. P.; Thornton, J. W.; Dickinson, B. C., Contingency and chance erase necessity in the experimental evolution of ancestral proteins. Elife 2021, 10.

95. Gibson, D. G.; Young, L.; Chuang, R. Y.; Venter, J. C.; Hutchison, C. A., 3rd; Smith, H. O., Enzymatic assembly of DNA molecules up to several hundred kilobases. Nat Methods 2009, 6 (5), 343-5. 